\title{
Design, Synthesis, and Evaluation of Novel 3-, 4-substituted, and 3,4-di Substituted Quinazoline Derivatives as Antimicrobial Agents
}

\author{
FARAG A. EL-ESSAWY ${ }^{1,2 *}$, ABDULRAHMAN I. ALHARTHI ${ }^{3}$, MSHARI A. ALOTAIBI ${ }^{3}$, \\ NANCY E. WAHBA ${ }^{2}$, NADER M. BOSHTA ${ }^{2}$ \\ ${ }^{1}$ Preparatory Year Deanship, Basic Science Department, Prince Sattam Bin Abdulaziz University, 151 Alkharj 11942, \\ KSA \\ ${ }^{2}$ Chemistry Department, Faculty of Science, Menoufia University, Shebin El-Koam, Egypt \\ ${ }^{3}$ Department of Chemistry, Collage of Science and Humanities, Prince Sattam Bin Abdulaziz University, 83Alkharj \\ 11942, KSA
}

\begin{abstract}
A novel series of 3-, 4-substituted, and 3,4-di substituted quinazoline derivatives were prepared via various cyclized regents and most of the newly prepared compounds evaluated for their antimicrobial activities in vitro against Gram-positive, Gram-negative bacterial strains and fungi strains. The structures of the quinazoline derivatives have been confirmed using spectroscopic analyses (IR, NMR, and EI-MS). Some of the synthesized derivatives displayed a moderate antimicrobial activity in comparison with reference drugs, for example compounds 13d, 15a, 17b, 18b, 18d, 25, and 29a-c. Among the synthesized compounds, the pyrimidoqunazoline derivative $6 \mathrm{c}$ elicited the highest activity.
\end{abstract}

Keywords: Quniazoline, imidazoline, pyrazolo, Trazolo, carbamide, antimicrobial

In the recent years, more and more interest has been focused on nitrogen heterocyclic systems and their applications. For example, the pharmaceutical reports reveal that nitrogen heterocycles are widely described as drug fragments. Among various nitrogen heterocyclic systems, quinazoline is one of the most prevalent heterocyclic rings found in the top 25 most frequent nitrogen heterocycles in U.S. FDA approved drug [1]. Moreover, it has been intensively studied due to their employment as building blocks in drug discovery showed good pharmacokinetics properties. Moreover, the ability to support a variable number of derivatives having six positions that can be substituted subsequently support the SAR study. In addition, the most of quinazoline derivatives having various biological activities including antimicrobial [2], analgesic and anti-inflammatory [3], anti-convulslant [4], anti-cancer [5], and anti-tubercular [6] activities. In addition, quinazoline is a core structure subunit in a variety of bioactive natural products $[7,8]$. Quinazoline derivatives have been reported to possess significant activity as antihypertensive [9], antifibrillatory, choleretic, antiphlogistic [10], antimitotic [11], antifungal [12] and anticonvulsant agents [13].

On the other hand, Pyrimidine, Triazole and pyrazole scaffolds attracted medicinal chemistry very much due to their biological and chemotherapeutic importance [14-23]. On this context and our research interest in the area of drug discovery as well as the synthesis of novel heterocyclic systems, [24-27] we became interested in the design and synthesis a series of novel quinazoline derivatives. Wherein the active pharmacophore triazole, pyrazole, pyrimidine heterocycles and others have been attached (fused/linkage) at the 3- and/or 4- positions of the quinazoline ring with enhanced biological significance (fig. 1). In addition, develop new useful methods for synthesis known heterocyclic systems having quinazoline as a main moiety from different starting point. The molecular structure of the newly synthesized compounds was investigated based on the spectral techniques.

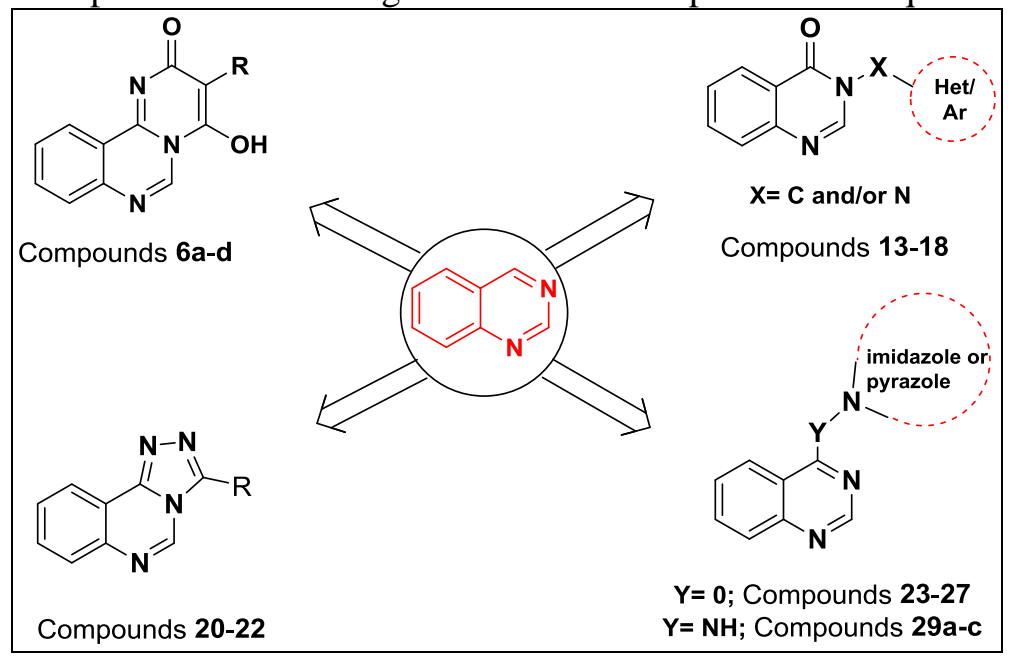

Fig. 1. Design of target compounds

*email: f.elessawy@psau.edu.sa 


\section{Experimental part}

Solvents and reagents were obtained from Acros (Geel, Belgium), Fluka (Taufkirchen, Germany) or Sigma (Steinheim, Germany). All melting points were measured on Electro thermal IA 9000 series digital melting Point apparatus. The IR spectra were recorded in potassium bromide discs on a Pye Unicam SP 3300 and Shimadzu FT IR 8101 PC infrared spectrophotometer. The NMR Spectra were recorded at $270 \mathrm{MHz}$ on a Varian Mercury VX-300 NMR spectrometer. ${ }^{1} \mathrm{H}$ NMR $(300 \mathrm{MHz})$ and ${ }^{13} \mathrm{C}$ NMR $(75 \mathrm{MHz})$ were run in deuterated chloroform $\left(\mathrm{CDCl}_{3}\right)$ or dimethylsulphoxide (DMSO- $d_{6}$ ). Chemical shifts were related to that of the solvent. Mass Spectra were recorded on a Shimadzu GCMS-QP1000 EX mass spectrometer at $70 \mathrm{eV}$. Elemental analyses were carried out at the Micro analytical Centre of Cairo University, Giza, Egypt. All reactions were followed by TLC (Silica gel, Aluminum Sheets 60 F254, Merck).

\section{4-Azidoquinazoline (3)}

A suspension of quinazolin-4-ol (1) $(1.46 \mathrm{~g}, 10 \mathrm{mmol})$ in $\mathrm{SOCl}_{2}(50 \mathrm{ml})$ and 2 drops DMF was heated under reflux until a clear solution was obtained $(20 \mathrm{~min})$, and then for a further $30 \mathrm{~min}$. The $\mathrm{SOCl}_{2}$ was removed under reduced pressure, and the residue was dissolved in $\mathrm{CH}_{2} \mathrm{Cl}_{2}$ and washed with aqueous $\mathrm{Na}_{2} \mathrm{CO}_{3}$. The solvent was dried and removed to give crude 4-chloroquinazoline (2) which was dissolved in DMF $(10 \mathrm{ml})$ and then sodium azide $(0.65 \mathrm{~g}$, $10 \mathrm{mmol}$ ) was added. The reaction mixture was stirred, at room temperature, for $3 \mathrm{hr}$ and the reaction mixture was poured into a cold water, the precipitate was filtered, dried, and recrystalized from ethanol. White solid, yield $1.20 \mathrm{~g}$ (71\%), mp 198-199 ${ }^{\circ} \mathrm{C}$; IR (KBr) $v: 2220\left(\mathrm{~N}_{3}\right), 1664(\mathrm{C}=\mathrm{N}) \mathrm{cm}^{-1}$; ${ }^{1} \mathrm{H}$ NMR (DMSO- $\left.d_{6}, 300 \mathrm{MHz}\right) \delta: 7.50-8.70(\mathrm{~m}$, $4 \mathrm{H}, \mathrm{ArH}), 9.50(\mathrm{~s}, 1 \mathrm{H}, \mathrm{H}-2, \mathrm{ArH}) \mathrm{ppm} ;{ }^{13} \mathrm{C}$ NMR (DMSO-d $\left.6,75 \mathrm{MHz}\right) \delta: 115.57,124.75,129.32,130.53,133.75$, 134.33, 142.85, $148.64(\mathrm{ArC}) \mathrm{ppm}$; MS (EI, $\mathrm{m} / z, 70 \mathrm{eV})$ : Calcd.= 171.05. Found $=171\left[\mathrm{M}^{+}\right]$; Anal. Calcd. for $\mathrm{C}_{8} \mathrm{H}_{5} \mathrm{~N}_{5}$ (171.16): C, 56.14; H, 2.94; N, 40.92. Found: C, 56.05; H, 2.64; N, $40.69 \%$.

\section{4-[(Triphenylphosphoranylidene)amino]quinazoline (4)}

A solution of azidoquinazoline $3(0.68 \mathrm{~g}, 4 \mathrm{mmol})$ and triphenylphosphane $(1.05 \mathrm{~g}, 4 \mathrm{mmol})$ in 1,2dichlorobenzene $(10 \mathrm{~mL})$ was heated under reflux for $30 \mathrm{~min}$. On cooling, the resulting solid product was collected by filtration, dried and recrystallized from ethanol to afford the colorless product, yield $1.11 \mathrm{~g}(69 \%)$, mp 196-198 ${ }^{\circ} \mathrm{C}$; IR (KBr) v: $1609(\mathrm{C}=\mathrm{N}), 1568,1426(\mathrm{Ar} \mathrm{C}=\mathrm{C}) \mathrm{cm}^{-1} ;{ }^{1} \mathrm{H}$ NMR (DMSO- $\left.d_{6}, 300 \mathrm{MHz}\right) \delta: 7.25-7.56(\mathrm{~m}, 17 \mathrm{H}, \mathrm{ArH})$, $7.58(\mathrm{~d}, 1 \mathrm{H}, J=6.4 \mathrm{MHz}, \mathrm{ArH}), 7.64(\mathrm{~s}, 1 \mathrm{H}, \mathrm{ArH}), 8.67$ (d, $1 \mathrm{H}, J=6.4 \mathrm{MHz}, \mathrm{ArH}) \mathrm{ppm} ;{ }^{13} \mathrm{C}$ NMR (DMSO- $d_{6}, 75$ MHz) $\delta: 122.11,125.31,126.07,126.37,126.52,130.68,131.41,132.37,154.42,166.60$ (ArC) ppm; MS (EI, $m / z, 70$ $\mathrm{eV}$ ): Calcd. $=405.14$. Found $=405\left[\mathrm{M}^{+}\right]$; Anal. Calcd. for $\mathrm{C}_{26} \mathrm{H}_{20} \mathrm{~N}_{3} \mathrm{P}$ (405.44): C, 77.02; H, 4.97; N, 10.36. Found: C, $76.85 ; \mathrm{H}, 4.64 ; \mathrm{N}, 10.24 \%$.

\section{Quinazolin-4-amine (5)}

A solution of iminophosphorane $4(0.81 \mathrm{~g}, 2 \mathrm{mmol})$ in acetic acid $(10 \mathrm{~mL}, 80 \%)$ was heated under reflux until the iminophosphoran hydrolyzed to the aminoquinazoline $(3 \mathrm{~h})$. The solvent was then removed under reduced pressure and the resulting solid product was digested with ethanol, the product was collected by filteration, washed with ethanol, dried, and recrystallized from methanol to afford the white prisms, yield $0.22 \mathrm{~g}(76 \%), \mathrm{mp} 252-253{ }^{\circ} \mathrm{C}$; IR $(\mathrm{KBr}) v: 3275\left(\mathrm{NH}_{2}\right), 1687(\mathrm{C}=\mathrm{N}) \mathrm{cm}^{-1} ;{ }^{1} \mathrm{H}$ NMR (DMSO- $\left.d_{6}, 300 \mathrm{MHz}\right) \delta: 7.47-7.78(\mathrm{~m}, 3 \mathrm{H}, \mathrm{ArH}), 7.93$ (brs, $2 \mathrm{H}$, $\mathrm{NH}_{2}$ ), 8.22 (d, $\left.1 \mathrm{H}, J=8 \mathrm{MHz}, \mathrm{H}-8, \mathrm{ArH}\right), 8.41$ (s, $1 \mathrm{H}, \mathrm{H}-2$, ArH) ppm; ${ }^{13} \mathrm{C}$ NMR (DMSO-d $\left.6,75 \mathrm{MHz}\right) \delta: 114.22$, 123.26, 125.61, 128.73, 132.06, 148.77, 155.12, 161.87 (ArC) ppm; MS (EI, m/z, $70 \mathrm{eV})$ : Calcd.= 145.06. Found= 145 [M+'] Anal. Calcd. for $\mathrm{C}_{8} \mathrm{H}_{7} \mathrm{~N}_{3}$ (145.17): C, 66.19; H, 4.86; N, 28.95. Found: C, 66.09, H, 4.59, N, $28.74 \%$.

\section{General procedure for the reaction aminoquinazoline with malonate derivatives $6 a-d$ :}

A mixture of 4-aminoquiazoline $(5)(0.14 \mathrm{~g}, 1 \mathrm{mmol})$ and the appropriate malonic ester $(1 \mathrm{mmol})$ in diphenyl ether $(5 \mathrm{ml})$ was refluxed in an oil bath for 20-40 min., using a short air condenser to remove the liberated ethanol. After cooling, the reaction mixture was triturated with diethyl ether, and the obtained precipitate was filtered off, washed with ether, dried, and recrystallized from methanol to afford 6a-d.

\section{4-Hydroxy-3-methyl-2H-pyrimido[1,2-c]quinazolin-2-one (6a)}

Pale yellow crystals, yield $0.16 \mathrm{~g}(76 \%), \mathrm{mp} 270-272{ }^{\circ} \mathrm{C}$; IR (KBr) v: 3330-3290 (OH), $1665(\mathrm{C}=\mathrm{O}) \mathrm{cm}^{-1}$; ${ }^{1} \mathrm{H}$ NMR (DMSO- $\left.d_{6}, 300 \mathrm{MHz}\right) \delta: 1.94\left(\mathrm{~s}, 3 \mathrm{H}, \mathrm{CH}_{3}\right), 7.76-8.05(\mathrm{~m}, 4 \mathrm{H}, \mathrm{ArH}), 9.31(\mathrm{~s}, 1 \mathrm{H}, \mathrm{H}-6, \mathrm{ArH}), 12.09$ (brs, 1H, OH) ppm; ${ }^{13} \mathrm{C}$ NMR (DMSO- $\left.d_{6}, 75 \mathrm{MHz}\right) \delta: 8.57\left(\mathrm{CH}_{3}\right), 94.51,112.28,119.52,120.09,125.03,128.11,128.88,136.22$,

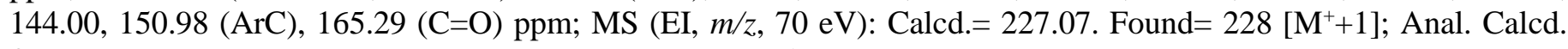
for $\mathrm{C}_{12} \mathrm{H}_{9} \mathrm{~N}_{3} \mathrm{O}_{2}$ (227.22): C, 63.43; H, 3.99; $\mathrm{N}, 18.49$. Found: $\mathrm{C}, 63.29 ; \mathrm{H}, 3.61 ; \mathrm{N}, 18.54 \%$.

\section{3-Ethyl-4-hydroxy-2H-pyrimido[1,2-c]quinazolin-2-one (6b)}

Pale Yellow powder, yield $0.17 \mathrm{~g}(74 \%), \mathrm{mp} 269-270{ }^{\circ} \mathrm{C}$; IR $(\mathrm{KBr}) v$ : 3350-3150 (OH), $1674(\mathrm{C}=\mathrm{O}) \mathrm{cm}^{-1} ;{ }^{1} \mathrm{H}$ NMR (DMSO- $\left.d_{6}, 300 \mathrm{MHz}\right) \delta: 1.04\left(\mathrm{t}, 3 \mathrm{H}, J=7.2 \mathrm{MHz}, \mathrm{CH}_{3} \mathrm{CH}_{2}\right.$ ), $3.42\left(\mathrm{q}, 2 \mathrm{H}, J_{l}=6.8, J_{2}=14.0 \mathrm{MHz}, \mathrm{CH}_{3} \mathrm{CH}_{2}\right.$ ), 
7.72-8.79 (m, 4H, ArH), 9.33 (s, 1H, H-6, ArH), 9.78 (brs, $1 \mathrm{H}, \mathrm{OH}) \mathrm{ppm} ;{ }^{13} \mathrm{C}$ NMR (DMSO- $\left.d_{6}, 75 \mathrm{MHz}\right) \delta: 12.53$ $\left(\mathrm{CH}_{3} \mathrm{CH}_{2}\right), 16.41\left(\mathrm{CH}_{3} \mathrm{CH}_{2}\right), 100.64,119.75,120.09,124.93,127.79,128.06,134.21,138.92,144.07,146.95(\mathrm{ArC})$, $165.08(\mathrm{C}=\mathrm{O}) \mathrm{ppm}$; MS (EI, $m / z, 70 \mathrm{eV})$ : Calcd.= 241.09. Found $=241\left[\mathrm{M}^{+}\right]$; Anal Calcd. for $\mathrm{C}_{13} \mathrm{H}_{11} \mathrm{~N}_{3} \mathrm{O}_{2}(241.25): \mathrm{C}$, 64.72; H, 4.60; N, 17.42. Found: C, 64.61; H, 4.41; N, $17.59 \%$.

\section{3-Butyl-4-hydroxy-2H-pyrimido[1,2-c]quinazolin-2-one (6c)}

Yellow crystals, yield $0.23 \mathrm{~g}(92 \%), \mathrm{mp} 281-282{ }^{\circ} \mathrm{C}$; IR $(\mathrm{KBr}) v: 3300-3185(\mathrm{OH}), 1645(\mathrm{C}=\mathrm{O}) \mathrm{cm}^{-1} ;{ }^{1} \mathrm{H}$ NMR (DMSO- $\left.d_{6}, 300 \mathrm{MHz}\right) \delta: 0.89\left(\mathrm{t}, 3 \mathrm{H}, J=7.4 \mathrm{MHz}, \mathrm{CH}_{3}\left(\mathrm{CH}_{2}\right)_{3}\right), 1.04\left(\mathrm{t}, 2 \mathrm{H}, J=7.2 \mathrm{MHz}, \mathrm{CH}_{2} \mathrm{CH}_{2} \mathrm{CH}_{2} \mathrm{CH}_{3}\right), 1.28-$ $1.51\left(\mathrm{~m}, 4 \mathrm{H}, \mathrm{CH}_{2} \mathrm{CH}_{2} \mathrm{CH}_{2} \mathrm{CH}_{3}\right), 7.75-8.61(\mathrm{~m}, 4 \mathrm{H}, \mathrm{ArH}), 9.33$ (s, 1H, H-6, ArH), 12.02 (brs, $\left.1 \mathrm{H}, \mathrm{OH}\right) \mathrm{ppm} ;{ }^{13} \mathrm{C}$ NMR (DMSO-d $6,75 \mathrm{MHz}) \delta: 13.93\left(\mathrm{CH}_{3}\right), 22.09,22.67,29.79\left(3 \mathrm{CH}_{2}\right), 99.34,120.09,124.91,127.83,128.90,134.21$, 138.04, 144.10, 146.95, 159.25 (ArC), $165.33(\mathrm{C}=\mathrm{O}) \mathrm{ppm}$; MS (EI, $\mathrm{m} / z, 70 \mathrm{eV})$ : Calcd.= 269.12. Found $=269\left[\mathrm{M}^{+}\right]$; Anal. Calcd. for $\mathrm{C}_{15} \mathrm{H}_{15} \mathrm{~N}_{3} \mathrm{O}_{2}$ (269.30): C, 66.90; H, 5.61; N, 15.60. Found: C, 66.49; H, 5.78; N, $15.70 \%$.

\section{4-Hydroxy-3-phenyl-2H-pyrimido[1,2-c]quinazolin-2-one (6d)}

Yellow powder, yield $0.18 \mathrm{~g}(67 \%)$, mp 293-294 ${ }^{\circ} \mathrm{C}$; IR $(\mathrm{KBr}) v: 3450-3300(\mathrm{OH}), 1676(\mathrm{C}=\mathrm{O}) \mathrm{cm}^{-1} ;{ }^{1} \mathrm{H}$ NMR (DMSO- $\left.d_{6}, 300 \mathrm{MHz}\right) \delta$ : 7.25-8.66 (s, 9H, Qui, Ph, ArH), 9.37 (s, $\left.1 \mathrm{H}, \mathrm{H}-6, \mathrm{ArH}\right), 12.37$ (brs, $\left.1 \mathrm{H}, \mathrm{OH}\right) \mathrm{ppm} ;{ }^{13} \mathrm{C}$ NMR $\left(\mathrm{DMSO}-d_{6}, 75 \mathrm{MHz}\right) \delta$ : 99.44, 119.85, 125.16, 127.41, 127.46, 127.53, 127.88, 129.04, 130.59, 130.66, 132.45, 134.64, 138.18, 144.43, 147.90, $158.48(\mathrm{ArC}), 164.85(\mathrm{C}=\mathrm{O})$ ppm; MS (EI, $\mathrm{m} / \mathrm{z}, 70 \mathrm{eV}):$ Calcd $=289.09$. Found $=289$ $\left[\mathrm{M}^{+}\right]$; Anal. Calcd. for $\mathrm{C}_{17} \mathrm{H}_{11} \mathrm{~N}_{3} \mathrm{O}_{2}$ (289.29): C, 70.58; H, 3.83; N, 14.53. Found: C, 70.33; H, 3.19; N, $14.42 \%$.

\section{2-(4-oxoquinazolin-3(4H)-yl)acetyl azide (10)}

Method A: To a cooled to $0-5{ }^{\circ} \mathrm{C}$ temperature suspension of hydrazide $(1 \mathrm{~g}, 4.6 \mathrm{mmol})$, water $(14 \mathrm{ml})$ and conc.hydrochloric acid $(4 \mathrm{~mL})$, a solution of sodium nitrite $(1.5 \mathrm{~g}, 23 \mathrm{mmol})$ in water $(9 \mathrm{ml})$ was added dropwise under stirring, the reaction mixture was stirred at this temperature for $1 \mathrm{~h}$. the precipitate was collected by filtration, washed with cold water and dried.

Method B: A solution of hydrazide $(1 \mathrm{~g}, 4.6 \mathrm{mmol})$ in $50 \%$ Acetic acid $(37 \mathrm{ml})$ was cooled to $0-5{ }^{\circ} \mathrm{C}$ and a solution of sodium nitrite $(0.4 \mathrm{~g}, 5.3 \mathrm{mmol})$ in water $(1 \mathrm{~mL})$ was added dropwise with vigorous stirring at such a rate that the reaction temperature did not exceed $5{ }^{\circ} \mathrm{C}$. The reaction mixture was stirred at the same temperature for $1.5 \mathrm{~h}$, the precipitate was filtered off and dried.

White solid, yield $0.85 \mathrm{~g}$ and $0.7 \mathrm{~g}(81 \%)$ and $(67 \%)$ respectively, mp 158-160 ${ }^{\circ} \mathrm{C}$; $\mathrm{IR}(\mathrm{KBr})$ v: $2152\left(\mathrm{~N}_{3}\right), 1720$, $1673(2 \mathrm{C}=\mathrm{O}) \mathrm{cm}^{-1} ;{ }^{1} \mathrm{H}$ NMR (DMSO- $\left.d_{6}, 500 \mathrm{MHz}\right) \delta: 4.70\left(\mathrm{~s}, 2 \mathrm{H}, \mathrm{CH}_{2}\right), 7.57(\mathrm{~m}, 1 \mathrm{H}, \mathrm{ArH}), 7.71(\mathrm{~d}, 1 \mathrm{H}, \operatorname{ArH}), 7.84$ $(\mathrm{m}, 1 \mathrm{H}, \mathrm{ArH}), 8.17(\mathrm{~d}, 1 \mathrm{H}, \mathrm{ArH}), 8.34(\mathrm{~s}, 1 \mathrm{H}, \mathrm{ArH}) \mathrm{ppm}$; MS $(\mathrm{EI}, \mathrm{m} / z, 70 \mathrm{eV})$ : Calcd.= 229.06. Found= 229.01 $\left[\mathrm{M}^{+}\right]$.

\section{3-aminoquinazolin-4(3H)-one (11)}

Method A: A mixture of $\mathbf{8}(1 \mathrm{~g}, 4.4 \mathrm{mmol})$ and hydrazine hydrate $(1.1 \mathrm{~g}, 22 \mathrm{mmol})$ in $(10 \mathrm{ml})$ absolute ethanol was heated under reflux for $24 \mathrm{~h}$. The reaction mixture was cooled, filtered off and dried to give 11.

Method B: A mixture of $7(1 \mathrm{~g}, 6.9 \mathrm{mmol})$ and hydrazine hydrate $(0.5 \mathrm{~g}, 10.4 \mathrm{mmol})$ in $(10 \mathrm{~mL})$ absolute ethanol was heated under reflux for $12 \mathrm{~h}$. The odour of ammonia must be finished. The reaction mixture was cooled and then poured onto ice water, filtered and dried to give 11 as a white solid, yield $0.5 \mathrm{~g}$ and $1 \mathrm{~g} \mathrm{(72 \% )}$ and (91\%) respectively, mp 188-190 ${ }^{\circ} \mathrm{C}$; IR (KBr) v: 3298-3172 $\left(\mathrm{NH}_{2}\right), 1682(\mathrm{C}=\mathrm{O}) \mathrm{cm}^{-1} ;{ }^{1} \mathrm{H}$ NMR (DMSO- $\left.d_{6}, 500 \mathrm{MHz}\right) \delta: 5.86(\mathrm{~s}, 2 \mathrm{H}$, $\left.\mathrm{NH}_{2}\right), 7.55(\mathrm{~m}, 1 \mathrm{H}, \mathrm{ArH}), 7.69(\mathrm{~m}, 1 \mathrm{H}, \mathrm{ArH}), 7.82(\mathrm{~m}, 1 \mathrm{H}, \mathrm{ArH}), 8.18(\mathrm{~d}, 1 \mathrm{H}, \mathrm{ArH}), 8.30(\mathrm{~s}, 1 \mathrm{H}, \mathrm{ArH}) \mathrm{ppm} ;{ }^{13} \mathrm{C} \mathrm{NMR}$ (DMSO- $\left.d_{6}, 75 \mathrm{MHz}\right) \delta: 121.35,125.83,126.85,127.24,133.95,147.61,148.30$ (ArC), 160.16 (C=O) ppm; MS (EI, $\mathrm{m} / \mathrm{z}, 70 \mathrm{eV})$ : Calcd. $=161.06$. Found $=161.21\left[\mathrm{M}^{+}\right], 162.16\left[\mathrm{M}^{+}+1\right]$.

\section{3-hydrazinylquinazolin-4(3H)-one (12)}

A mixture of $11(3 \mathrm{~g}, 18.7 \mathrm{mmol})$ and aqueous solution of $\mathrm{NaNO}_{2}(9 \mathrm{~g}(7.5 \mathrm{~mL}) 131 \mathrm{mmol})$ were successively added to aqueous hydrochloric acid $(12 \mathrm{ml})$ maintaining the temperature between $\left(0\right.$ and $\left.5{ }^{\circ} \mathrm{C}\right)$. The reaction mixture was stirred for $(2 \mathrm{~h})$. Alkalized to $\mathrm{PH}(6-7)$ with $12 \%$ sodium carbonate solution, the resulting diazonium salt was added dropwise to sodium sulfite solution $(25 \mathrm{~g}(45.8 \mathrm{ml}) 201 \mathrm{mmol})$ below $0{ }^{\circ} \mathrm{C}$. Then the mixture was stirred at room temperature for $1 \mathrm{~h}$, concentrated hydrochloric acid $(29 \mathrm{ml})$ was added slowly and heated to $100{ }^{\circ} \mathrm{C}$ for $3 \mathrm{~h}$. the mixture was then cooled and filtered.

White solid, yield $2.5 \mathrm{~g}(76 \%), \mathrm{mp} 204-206{ }^{\circ} \mathrm{C} ;{ }^{1} \mathrm{H}$ NMR (DMSO- $\left.d_{6}, 500 \mathrm{MHz}\right) \delta: 7.53(\mathrm{~m}, 1 \mathrm{H}, \mathrm{ArH}), 7.67(\mathrm{~d}$, $1 \mathrm{H}, \mathrm{ArH}), 7.82(\mathrm{~m}, 1 \mathrm{H}, \mathrm{ArH}), 8.13(\mathrm{~m}, 2 \mathrm{H}, \mathrm{ArH}) \mathrm{ppm} ;{ }^{13} \mathrm{C}$ NMR (DMSO-d $\left.6,75 \mathrm{MHz}\right) \delta: 122.59,125.79,126.68$, 127.12, 134.25, 145.41, $148.69(\mathrm{ArC}), 160.74(\mathrm{C}=\mathrm{O}) \mathrm{ppm}$; MS (EI, $\mathrm{m} / \mathrm{z}, 70 \mathrm{eV}):$ Calcd.= 176.07. Found $=176.34$ $\left[\mathrm{M}^{+}\right]$.

\section{General procedure for the preparation of compounds 13a-f:}

A mixture of $11(0.2 \mathrm{~g}, 1.3 \mathrm{mmol})$ and aldehyde $(1.3 \mathrm{mmol})$ in $(2 \mathrm{~mL})$ absolute ethanol, (5 drops) of piperidine or acetic acid was added. The reaction mixture was heated under reflux for $8 \mathrm{~h}$. The formed solid was filtered off and dried. 
(E)-3-[(2-bromobenzylidene)amino]quinazolin-4(3H)-one (13a)

White solid, yield $0.3 \mathrm{~g}(75 \%), \mathrm{mp} 158-160{ }^{\circ} \mathrm{C} ;{ }^{1} \mathrm{H}$ NMR (DMSO- $\left.d_{6}, 500 \mathrm{MHz}\right) \delta: 7.42(\mathrm{~m}, 2 \mathrm{H}, \mathrm{ArH}), 7.62(\mathrm{~m}$, 1H, ArH), $7.76(\mathrm{~m}, 1 \mathrm{H}, \operatorname{ArH}), 7.90(\mathrm{~m}, 1 \mathrm{H}, \mathrm{ArH}), 8.03(\mathrm{~m}, 2 \mathrm{H}, \operatorname{ArH}), 8.25(\mathrm{~d}, 1 \mathrm{H}, \operatorname{ArH}), 8.57(\mathrm{~s}, 1 \mathrm{H}, \operatorname{ArH}), 9.31(\mathrm{~s}$, $1 \mathrm{H}, \mathrm{N}=\mathrm{CH}) \mathrm{ppm}$; MS (EI, $\mathrm{m} / z, 70 \mathrm{eV})$ : Calcd.= 327. Found=327.9 $\left[\mathrm{M}^{+}\right], 329.9\left[\mathrm{M}^{+}+2\right]$.

(E)-3-[(4-fluorobenzylidene)amino]quinazolin-4(3H)-one (13b)

Pale yellow solid, yield $0.3 \mathrm{~g}(91 \%)$, mp 148-150 ${ }^{\circ} \mathrm{C} ;{ }^{1} \mathrm{H}$ NMR (DMSO- $\left.d_{6}, 500 \mathrm{MHz}\right) \delta: 7.54(\mathrm{~m}, 2 \mathrm{H}, \mathrm{ArH}), 7.64$ $(\mathrm{m}, 1 \mathrm{H}, \mathrm{ArH}), 7.76(\mathrm{~m}, 1 \mathrm{H}, \mathrm{ArH}), 7.82(\mathrm{~d}, 1 \mathrm{H}, \mathrm{ArH}), 7.89(\mathrm{~m}, 1 \mathrm{H}, \mathrm{ArH}), 8.18(\mathrm{~m}, 1 \mathrm{H}, \mathrm{ArH}), 8.27(\mathrm{~d}, 1 \mathrm{H}, \operatorname{ArH}), 8.58$ $(\mathrm{s}, 1 \mathrm{H}, \mathrm{ArH}), 9.88(\mathrm{~s}, 1 \mathrm{H}, \mathrm{N}=\mathrm{CH}) \mathrm{ppm} ;{ }^{13} \mathrm{C}$ NMR (DMSO-d 6 , $\left.75 \mathrm{MHz}\right) \delta: 122.33,125.25,126.68,127.36,127.47$, $128.08,128.32,131.78,133.50,133.91,134.66,146.26,146.28,158.50(\mathrm{ArC}$ and $\mathrm{N}=\mathrm{C}), 161.60(\mathrm{C}=\mathrm{O}) \mathrm{ppm}$; MS (EI, $\mathrm{m} / \mathrm{z}, 70 \mathrm{eV})$ : Calcd. $=267.08$. Found $=267.9\left[\mathrm{M}^{+}\right]$.

(E)-3-[(4-chlorobenzylidene)amino]quinazolin-4(3H)-one (13c)

White solid, yield $0.3 \mathrm{~g}(86 \%), \mathrm{mp} 168-170{ }^{\circ} \mathrm{C} ;{ }^{1} \mathrm{H}$ NMR (DMSO- $\left.d_{6}, 500 \mathrm{MHz}\right) \delta: 7.61(\mathrm{~m}, 2 \mathrm{H}, \mathrm{ArH}), 7.66(\mathrm{~d}, 1 \mathrm{H}$, $\operatorname{ArH}), 7.76(\mathrm{~d}, 1 \mathrm{H}, \mathrm{ArH}), 7.89(\mathrm{~m}, 1 \mathrm{H}, \mathrm{ArH}), 7.96(\mathrm{~d}, 2 \mathrm{H}, \mathrm{ArH}), 8.24(\mathrm{~d}, 1 \mathrm{H}, \mathrm{ArH}), 8.58(\mathrm{~s}, 1 \mathrm{H}, \operatorname{ArH}), 9.35(\mathrm{~s}, 1 \mathrm{H}$, $\mathrm{N}=C H$ ) ppm; ${ }^{13} \mathrm{C}$ NMR (DMSO- $\left.d_{6}, 75 \mathrm{MHz}\right) \delta: 122.27,126.66,127.47,127.54,131.56,134.67,137.19,145.57$, 145.58, 146.61, $157.70(\mathrm{ArC}$ and $\mathrm{N}=\mathrm{C}), 164.09(\mathrm{C}=\mathrm{O}) \mathrm{ppm}$; MS (EI, $\mathrm{m} / \mathrm{z}, 70 \mathrm{eV})$ : Clacd. $=283.05$. Found $=283.09$ $\left[\mathrm{M}^{+}\right]$.

(E)-3-(benzylideneamino)quinazolin-4(3H)-one (13d)

White solid, yield $0.25 \mathrm{~g}(83 \%)$, mp 118-120 ${ }^{\circ} \mathrm{C} ;{ }^{1} \mathrm{H}$ NMR (DMSO-d $\left.6,500 \mathrm{MHz}\right) \delta: 7.6(\mathrm{~m}, 4 \mathrm{H}, \mathrm{ArH}), 7.7(\mathrm{~d}, 1 \mathrm{H}$, ArH), $7.8(\mathrm{~m}, 1 \mathrm{H}, \mathrm{ArH}), 7.9(\mathrm{~m}, 2 \mathrm{H}, \mathrm{ArH}), 8.24(\mathrm{~d}, 1 \mathrm{H}, \mathrm{ArH}), 8.58(\mathrm{~s}, 1 \mathrm{H}, \mathrm{ArH}), 9.3(\mathrm{~s}, 1 \mathrm{H}, \mathrm{N}=C H) \mathrm{ppm} ;{ }^{13} \mathrm{C}$ NMR (DMSO- $\left.d_{6}, 75 \mathrm{MHz}\right) \delta: 122.42,126.73,127.56,128.84,129.27,129.29,132.70,134.67,145.65,146.81,157.75$ (ArC and $\mathrm{N}=\mathrm{C}), 165.89(\mathrm{C}=\mathrm{O}) \mathrm{ppm}$; MS (EI, $\mathrm{m} / z, 70 \mathrm{eV})$ : Calcd.=249.09. Found=249.44 $\left[\mathrm{M}^{+}\right]$.

(E)-3-[(4-methoxybenzylidene)amino]quinazolin-4(3H)-one (13e)

White solid, yield $0.3 \mathrm{~g}(88 \%)$, mp 128-130 ${ }^{\circ} \mathrm{C} ;{ }^{1} \mathrm{H}$ NMR (DMSO- $\left.d_{6}, 500 \mathrm{MHz}\right) \delta: 3.86\left(\mathrm{~s}, 3 \mathrm{H}, \mathrm{CH}_{3}\right), 7.13(\mathrm{~d}, 2 \mathrm{H}$, ArH), $7.61(\mathrm{~m}, 1 \mathrm{H}, \mathrm{ArH}), 7.74(\mathrm{~m}, 1 \mathrm{H}, \mathrm{ArH}), 7.90(\mathrm{~m}, 3 \mathrm{H}, \mathrm{ArH}), 8.24(\mathrm{~m}, 1 \mathrm{H}, \mathrm{ArH}), 8.53(\mathrm{~s}, 1 \mathrm{H}, \mathrm{ArH}), 9.13(\mathrm{~s}, 1 \mathrm{H}$, $\mathrm{N}=C H) \mathrm{ppm}$; MS $(\mathrm{EI}, \mathrm{m} / z, 70 \mathrm{eV})$ : Calcd. $=279.10$. Found $=279.06\left[\mathrm{M}^{+}\right]$.

(E)-3-[(2-hydroxybenzylidene)amino]quinazolin-4(3H)-one (13f)

White solid, yield $0.29 \mathrm{~g}(91 \%), \mathrm{mp} 200-202{ }^{\circ} \mathrm{C} ;{ }^{1} \mathrm{H}$ NMR (DMSO- $\left.d_{6}, 500 \mathrm{MHz}\right) \delta: 6.98(\mathrm{~m}, 1 \mathrm{H}, \mathrm{ArH}), 7.02(\mathrm{~m}$, 1H, ArH), $7.48(\mathrm{~m}, 1 \mathrm{H}, \mathrm{ArH}), 7.61(\mathrm{~m}, 1 \mathrm{H}, \mathrm{ArH}), 7.76(\mathrm{~m}, 1 \mathrm{H}, \mathrm{ArH}), 7.86(\mathrm{~m}, 2 \mathrm{H}, \mathrm{ArH}), 8.24(\mathrm{~d}, 1 \mathrm{H}, \mathrm{ArH}), 8.58(\mathrm{~s}, 1$ $\mathrm{H}, \mathrm{ArH}$ ), $9.46(\mathrm{~s}, 1 \mathrm{H}, \mathrm{N}=\mathrm{CH}), 10.5$ (brs, $1 \mathrm{H}, \mathrm{OH}) \mathrm{ppm} ;{ }^{13} \mathrm{C}$ NMR (DMSO-d 6 , $\left.75 \mathrm{MHz}\right) \delta: 116.71,118.21,119.54$, $122.25,126.52,127.28,127.35,128.43,134.07,134.39,145.34,146.61,157.55,158.45$ (ArC and $\mathrm{N}=\mathrm{C}$ ), 163.16 $(\mathrm{C}=\mathrm{O}) \mathrm{ppm}$; MS (EI, $\mathrm{m} / \mathrm{z}, 70 \mathrm{eV})$ : Calcd.= 265.09. Found $=265.33\left[\mathrm{M}^{+}\right], 267.60\left[\mathrm{M}^{+}+2\right]$.

$\mathrm{N}$-(4-oxoquinazolin-3(4H)-yl)acetamide (14a)

Method A: A suspension of $\mathbf{1 1}(0.1 \mathrm{~g}, 0.63 \mathrm{mmol})$ in acetic anhydride $(4 \mathrm{ml})$ was heated under reflux for $1 \mathrm{~h}$, the mixture was cooled and poured onto crushed ice. The product that separated out filtered off, washed with water and then dried.

Method B: A mixture of compound $11(0.5 \mathrm{~g}, 3.2 \mathrm{mmol})$ and acetyl bromide $(0.4 \mathrm{~g}, 3.2 \mathrm{mmol})$ in dry dioxane (32 $\mathrm{ml}$ ) was heated under reflux for $10 \mathrm{~h}$, the excess solvent was removed under reduced pressure. The product that separated out filtered off then dried.

Off white solid, yield $0.1 \mathrm{~g}$ and $0.38 \mathrm{~g}(83 \%)$ and $(60 \%)$ respectively, mp 198-200 ${ }^{\circ} \mathrm{C} ;{ }^{1} \mathrm{H}$ NMR (DMSO- $d_{6}, 400$ MHz) $\delta: 2.07\left(\mathrm{~s}, 3 \mathrm{H}, \mathrm{CH}_{3}\right), 7.58(\mathrm{~m}, 1 \mathrm{H}, \mathrm{ArH}), 7.73(\mathrm{~d}, 1 \mathrm{H}, \mathrm{ArH}), 7.88(\mathrm{~m}, 1 \mathrm{H}, \mathrm{ArH}), 8.17(\mathrm{~d}, 1 \mathrm{H}, \mathrm{ArH}), 8.22(\mathrm{~s}, 1 \mathrm{H}$, ArH), 11.24 (brs, 1H, NH) ppm; MS (EI, $m / z, 70 \mathrm{eV}$ ): Calcd.= 203.07. Found= $203.18\left[\mathrm{M}^{+}\right], 204\left[\mathrm{M}^{+}+1\right]$.

\section{$N$-(4-oxoquinazolin-3(4H)-yl)benzamide (14b)}

A mixture of compound $11(0.5 \mathrm{~g}, 3.2 \mathrm{mmol})$ and benzoyl chloride $(0.4 \mathrm{~g}, 3.2 \mathrm{mmol})$ in dry dioxane $(32 \mathrm{ml})$ was heated under reflux for $18 \mathrm{~h}$, the excess solvent was removed under reduced pressure. The product that separated out filtered off then dried.

White solid, yield $0.43 \mathrm{~g}(52 \%), \mathrm{mp} 190-192{ }^{\circ} \mathrm{C} ;{ }^{1} \mathrm{H}$ NMR (DMSO- $\left.d_{6}, 500 \mathrm{MHz}\right) \delta: 7.62(\mathrm{~m}, 2 \mathrm{H}, \mathrm{ArH}), 7.70(\mathrm{~m}$, 2H, ArH), $7.79(\mathrm{~m}, 1 \mathrm{H}, \mathrm{ArH}), 7.93(\mathrm{~m}, 1 \mathrm{H}, \mathrm{ArH}), 8.01(\mathrm{~m}, 2 \mathrm{H}, \mathrm{ArH}), 8.23(\mathrm{~d}, 1 \mathrm{H}, \mathrm{ArH}), 8.45(\mathrm{~s}, 1 \mathrm{H}, \mathrm{ArH}), 11.86$ (brs, $1 \mathrm{H}, \mathrm{NH}) \mathrm{ppm} ;{ }^{13} \mathrm{C}$ NMR (DMSO- $\left.d_{6}, 75 \mathrm{MHz}\right) \delta: 121.98,126.44,127.63,127.68,127.83,128.77,131.13$, 132.82, 135.09, 147.30, $149.08(\mathrm{ArC}), 158.59,166.31(2 \mathrm{C}=\mathrm{O}) \mathrm{ppm}$; MS $(\mathrm{EI}, \mathrm{m} / \mathrm{z}, 70 \mathrm{eV})$ : Calcd.= 265.09. Found= $265.9\left[\mathrm{M}^{+}\right]$. 
General procedure for the preparation of compounds 15a,b:

To a solution of $\mathbf{1 3 f}(0.2 \mathrm{~g}, 0.8 \mathrm{mmol})$ in $(2 \mathrm{~mL}) \mathrm{DMF}$, ethylbromoacetate and/ or chloroacetonitrile $(0.8 \mathrm{mmol})$ and potassium carbonate anhydrous $(0.8 \mathrm{mmol})$ was added. The reaction mixture was stirred at room temperature for $24 \mathrm{~h}$. Then poured onto ice water and the formed solid collected by filtration.

\section{Ethyl (E)-2-(2-(((4-oxoquinazolin-3(4H)-yl)imino)methyl) phenoxy) acetate (15a)}

Off white solid, yield $0.23 \mathrm{~g}(88 \%), \mathrm{mp} 122-124{ }^{\circ} \mathrm{C} ;{ }^{1} \mathrm{H}$ NMR (DMSO- $\left.d_{6}, 400 \mathrm{MHz}\right) \delta: 1.18$ (t, 3H, $\left.\mathrm{CH}_{3}\right), 4.16$ (q, $\left.2 \mathrm{H}, \mathrm{CH}_{3} \mathrm{CH}_{2}\right), 4.97\left(\mathrm{~s}, 2 \mathrm{H}, \mathrm{CH}_{2}\right), 7.12(\mathrm{~m}, 2 \mathrm{H}, \mathrm{ArH}), 7.57(\mathrm{~m}, 2 \mathrm{H}, \mathrm{ArH}), 7.74(\mathrm{~m}, 1 \mathrm{H}, \mathrm{ArH}), 7.87(\mathrm{~m}, 1 \mathrm{H}, \mathrm{ArH}), 8.04$ $(\mathrm{d}, 1 \mathrm{H}, \mathrm{ArH}), 8.23(\mathrm{~d}, 1 \mathrm{H}, \mathrm{ArH}), 8.53(\mathrm{~s}, 1 \mathrm{H}, \mathrm{ArH}), 9.66(\mathrm{~s}, 1 \mathrm{H}, \mathrm{N}=C H) \mathrm{ppm} ; \mathrm{MS}(\mathrm{EI}, \mathrm{m} / z, 70 \mathrm{eV})$ : Calcd.= 351.12. Found $=351.04\left[\mathrm{M}^{+}\right]$.

\section{(E)-2-(2-(((4-oxoquinazolin-3(4H)-yl)imino)methyl)phenoxy)aceto-nitrile (15b)}

Off white solid, yield $0.2 \mathrm{~g}(91 \%), \mathrm{mp} 118-120{ }^{\circ} \mathrm{C} ;{ }^{1} \mathrm{H}$ NMR (DMSO- $\left.d_{6}, 500 \mathrm{MHz}\right) \delta: 5.33\left(\mathrm{~s}, 2 \mathrm{H}, \mathrm{CH}_{2}\right), 7.27(\mathrm{~m}$, $1 \mathrm{H}, \mathrm{ArH}), 7.36(\mathrm{~m}, 1 \mathrm{H}, \mathrm{ArH}), 7.62(\mathrm{~m}, 1 \mathrm{H}, \mathrm{ArH}), 7.68(\mathrm{~m}, 1 \mathrm{H}, \mathrm{ArH}), 7.76(\mathrm{~m}, 1 \mathrm{H}, \mathrm{ArH}), 7.88(\mathrm{~m}, 1 \mathrm{H}, \mathrm{ArH}), 8.12(\mathrm{~d}$, $1 \mathrm{H}, \mathrm{ArH}), 8.25(\mathrm{~d}, 1 \mathrm{H}, \mathrm{ArH}), 8.55(\mathrm{~s}, 1 \mathrm{H}, \mathrm{ArH}), 9.64(\mathrm{~s}, 1 \mathrm{H}, \mathrm{N}=\mathrm{CH}) \mathrm{ppm} ;{ }^{13} \mathrm{C}$ NMR (DMSO-d, $\left.75 \mathrm{MHz}\right) \delta: 54.14$ $\left(\mathrm{CH}_{2}\right), 113.51,116.31,122.86,126.63,126.64,127.02,127.38,134.21,134.53,145.98,146.52,156.11,157.97(\mathrm{ArC}$ and $\mathrm{CN}), 159.51(\mathrm{C}=\mathrm{O}) \mathrm{ppm}$; MS $(\mathrm{EI}, \mathrm{m} / \mathrm{z}, 70 \mathrm{eV})$ : Calcd.= 304.10. Found $=305.2\left[\mathrm{M}^{+}+1\right]$.

\section{2-chloro- $N$-(4-oxoquinazolin-3(4H)-yl)acetamide (16)}

A solution of $11(1 \mathrm{~g}, 6.3 \mathrm{mmol})$ in $(10 \mathrm{~mL})$ DMF containing chloroacetyl chloride $(0.7 \mathrm{~g}, 6.3 \mathrm{mmol})$ was stirred at room temperature for $24 \mathrm{~h}$. The solution was poured onto ice and then the resulting solid was filtered and dried to give 16 as a white solid, yield $1.4 \mathrm{~g}(95 \%)$, mp $170-172{ }^{\circ} \mathrm{C}$; IR $(\mathrm{KBr})$ v: $3206(\mathrm{NH}), 1706,1668(2 \mathrm{C}=\mathrm{O}) \mathrm{cm}^{-1}$; ${ }^{1} \mathrm{H}$ NMR $\left(\mathrm{DMSO}-d_{6}, 500 \mathrm{MHz}\right) \delta: 4.42\left(\mathrm{~s}, 2 \mathrm{H}, \mathrm{CH}_{2}\right), 7.62(\mathrm{~m}, 1 \mathrm{H}, \mathrm{ArH}), 7.76(\mathrm{~d}, 1 \mathrm{H}, \mathrm{ArH}), 7.91(\mathrm{~m}, 1 \mathrm{H}, \operatorname{ArH}), 8.19(\mathrm{~d}, 1 \mathrm{H}$, $\mathrm{ArH}), 8.27(\mathrm{~s}, 1 \mathrm{H}, \mathrm{ArH}), 11.67(\mathrm{~s}, 1 \mathrm{H}, \mathrm{NH}) \mathrm{ppm} ;{ }^{13} \mathrm{C}$ NMR (DMSO- $\left.d_{6}, 75 \mathrm{MHz}\right) \delta: 40.74\left(\mathrm{CH}_{2}\right), 121.87,126.37$, 127.54, 127.62, 135.03, 147.08, 148.35 (ArC), 158.08, $166.30(2 \mathrm{C}=\mathrm{O}) \mathrm{ppm}$; MS (EI, $\mathrm{m} / z, 70 \mathrm{eV})$ : Calcd.= 237.03. Found $=237.33\left[\mathrm{M}^{+}\right]$.

General procedure for the preparation of compounds $17 a-c$ :

To a solution of $16(0.2 \mathrm{~g}, 0.7 \mathrm{mmol})$ in $(2 \mathrm{~mL})$ acetonitrile, piperidine and/ or diphenyl amine and/ or 2,5-dimethyl aniline $(0.7 \mathrm{mmol})$ and potassium carbonate anhydrous $(0.7 \mathrm{mmol})$ was added. The reaction mixture was stirred at room temperature for $24 \mathrm{~h}$. Then the formed solid collected by filtration.

\section{$N$-(4-oxoquinazolin-3(4H)-yl)-2-(piperidin-1-yl)acetamide (17a)}

Off white solid, yield $0.2 \mathrm{~g}(83 \%), \mathrm{mp}>360{ }^{\circ} \mathrm{C} ;{ }^{1} \mathrm{H}$ NMR (DMSO- $\left.d_{6}, 500 \mathrm{MHz}\right) \delta: 1.43-1.46$ (m, $2 \mathrm{H}, \mathrm{CH}_{2}$ ), 1.56-1.61 (m, 4H, $\left.\mathrm{CH}_{2}\right), 3.40-3.50\left(\mathrm{~m}, 4 \mathrm{H}, \mathrm{CH}_{2}\right), 4.92\left(\mathrm{~s}, 2 \mathrm{H}, \mathrm{COCH}_{2}\right), 7.56(\mathrm{~m}, 1 \mathrm{H}, \mathrm{ArH}), 7.70(\mathrm{~d}, 1 \mathrm{H}, \mathrm{ArH}), 7.84$ $(\mathrm{m}, 1 \mathrm{H}, \mathrm{ArH}), 8.15(\mathrm{~d}, 1 \mathrm{H}, \mathrm{ArH}), 8.24(\mathrm{~s}, 1 \mathrm{H}, \mathrm{ArH}) \mathrm{ppm} ;{ }^{13} \mathrm{C}$ NMR (DMSO- $\left.d_{6}, 75 \mathrm{MHz}\right) \delta: 23.85,25.13,25.83$, $45.19,46.55,62.78\left(6 \mathrm{CH}_{2}\right), 121.45,125.98,126.99,127.15,134.35,148.04,148.58(\mathrm{ArC}), 160.13,164.47(2 \mathrm{C}=\mathrm{O})$ ppm; MS (EI, $m / z, 70 \mathrm{eV})$ : Calcd.= 286.14. Found $=287.2\left[\mathrm{M}^{+}+1\right]$.

\section{2-(diphenylamino)- $N$-(4-oxoquinazolin-3(4H)-yl)acetamide (17b)}

Off white solid, yield $0.25 \mathrm{~g}(81 \%)$, mp $318-320{ }^{\circ} \mathrm{C} ;{ }^{1} \mathrm{H}$ NMR (DMSO- $\left.d_{6}, 500 \mathrm{MHz}\right) \delta: 3.8\left(\mathrm{~s}, 2 \mathrm{H}, \mathrm{CH}_{2}\right), 6.81(\mathrm{~m}$, $1 \mathrm{H}, \operatorname{ArH}), 7.06(\mathrm{~d}, J=8.53 \mathrm{MHz}, 1 \mathrm{H}, \mathrm{ArH}), 7.22(\mathrm{~m}, 1 \mathrm{H}, \mathrm{ArH}), 7.43(\mathrm{~m}, 2 \mathrm{H}, \mathrm{ArH}), 7.60(\mathrm{~m}, 2 \mathrm{H}, \operatorname{ArH}), 7.67(\mathrm{~m}, 3 \mathrm{H}$, ArH), 7.92 (s, 2H, ArH), 8.09 (m, 2H, ArH), 8.24 (d, $J=8.01 \mathrm{MHz}, 1 \mathrm{H}, \mathrm{ArH}) . \mathrm{ppm} ;{ }^{13} \mathrm{C}$ NMR (DMSO- $\left.d_{6}, 75 \mathrm{MHz}\right)$ $\delta: 45.74\left(\mathrm{CH}_{2}\right), 116.68,119.57,123.06,125.39,125.74,126.50,126.63,127.67,127.80,129.07,132.39,135.40$, 143.40, 147.33, 147.50, 147.64, 149.78, 158.44 (ArC), 162.74, $169.38(2 \mathrm{C}=\mathrm{O}) \mathrm{ppm}$. MS (EI, m/z, $70 \mathrm{eV})$ : Calcd.= 370.14. Found $=370.73\left[\mathrm{M}^{+}\right]$.

\section{2-[(2,5-dimethylphenyl)amino]-N-(4-oxoquinazolin-3(4H)-yl)acetamide (17c)}

Off white solid, yield $0.22 \mathrm{~g}(81 \%), \mathrm{mp} \mathrm{306-308}{ }^{\circ} \mathrm{C}$; ${ }^{1} \mathrm{H}$ NMR (DMSO- $\left.d_{6}, 500 \mathrm{MHz}\right) \delta: 2.48-2.51\left(\mathrm{~s}, 6 \mathrm{H}, 2 \mathrm{CH}_{3}\right.$ ), $3.8\left(\mathrm{~s}, 2 \mathrm{H}, \mathrm{CH}_{2}\right), 7.43(\mathrm{~m}, 1 \mathrm{H}, \mathrm{ArH}), 7.60(\mathrm{~m}, 1 \mathrm{H}, \mathrm{ArH}), 7.69(\mathrm{~m}, 2 \mathrm{H}, \mathrm{ArH}), 7.79(\mathrm{~m}, 1 \mathrm{H}, \mathrm{ArH}), 7.91(\mathrm{~s}, 1 \mathrm{H}, \operatorname{ArH})$, $8.09(\mathrm{~m}, 1 \mathrm{H}, \mathrm{ArH}), 8.24(\mathrm{~d}, 1 \mathrm{H}, \mathrm{ArH}) \mathrm{ppm}$. MS (EI, $\mathrm{m} / z, 70 \mathrm{eV})$ : Calcd.= 322.14. Found= $322.21\left[\mathrm{M}^{+}\right]$.

\section{General procedure for the preparation of compounds $18 a-j$ :}

To a solution of azide $\mathbf{1 0}(1 \mathrm{~g}, 4.4 \mathrm{mmol})$ in dry dioxane $(30 \mathrm{~mL})$ the corresponding secondary amine and/ or aniline (primary amine) $(4.4 \mathrm{mmol})$ was added. The reaction mixture was heated at reflux for $8 \mathrm{~h}$, then cooled to room temperature. The resultant precipitate was collected by filtration. 


\section{$N$-[(4-oxoquinazolin-3(4H)-yl)methyl]morpholine-4-carboxamide (18a)}

Pale yellow solid, yield $1.1 \mathrm{~g}(88 \%)$, mp $222-224{ }^{\circ} \mathrm{C} ;{ }^{1} \mathrm{H}$ NMR (DMSO- $\left.d_{6}, 300 \mathrm{MHz}\right) \delta: 3.44\left(\mathrm{~m}, 2 \mathrm{H}, \mathrm{CH}_{2}\right.$ ), 3.58 $\left(\mathrm{m}, 4 \mathrm{H}, \mathrm{CH}_{2}\right), 3.66\left(\mathrm{~m}, 2 \mathrm{H}, \mathrm{CH}_{2}\right), 4.94\left(\mathrm{~s}, 2 \mathrm{H}, \mathrm{CH}_{2}\right), 7.56(\mathrm{~m}, 1 \mathrm{H}, \mathrm{ArH}), 7.70(\mathrm{~d}, 1 \mathrm{H}, \mathrm{ArH}), 7.83(\mathrm{~m}, 1 \mathrm{H}, \operatorname{ArH}), 8.13$ $(\mathrm{d}, 1 \mathrm{H}, \mathrm{ArH}), 8.23(\mathrm{~s}, 1 \mathrm{H}, \mathrm{ArH}) \mathrm{ppm} ; \mathrm{MS}(\mathrm{EI}, \mathrm{m} / z, 70 \mathrm{eV})$ : Calcd.= 288.12. Found= $288.40\left[\mathrm{M}^{+}\right], 290.01\left[\mathrm{M}^{+}+1\right]$.

\section{$\mathrm{N}$-[(4-oxoquinazolin-3(4H)-yl)methyl]piperidine-1-carboxamide (18b)}

Pale yellow solid, yield $1 \mathrm{~g}(81 \%)$, mp 218-220 ${ }^{\circ} \mathrm{C} ;{ }^{1} \mathrm{H}$ NMR (DMSO- $\left.d_{6}, 500 \mathrm{MHz}\right) \delta: 1.44\left(\mathrm{~m}, 2 \mathrm{H}, \mathrm{CH}_{2}\right), 1.60(\mathrm{~m}$, $\left.4 \mathrm{H}, 2 \mathrm{CH}_{2}\right), 3.38\left(\mathrm{~m}, 2 \mathrm{H}, \mathrm{CH}_{2}\right), 3.42\left(\mathrm{~m}, 2 \mathrm{H}, \mathrm{CH}_{2}\right), 4.94\left(\mathrm{~s}, 2 \mathrm{H}, \mathrm{CH}_{2}\right), 7.54(\mathrm{~m}, 1 \mathrm{H}, \mathrm{ArH}), 7.69(\mathrm{~d}, 1 \mathrm{H}, \mathrm{ArH}), 7.83(\mathrm{~m}$, $1 \mathrm{H}, \mathrm{ArH}), 8.13(\mathrm{~d}, 1 \mathrm{H}, \mathrm{ArH}), 8.24(\mathrm{~s}, 1 \mathrm{H}, \mathrm{ArH}) \mathrm{ppm} ; \mathrm{MS}(\mathrm{EI}, \mathrm{m} / z, 70 \mathrm{eV})$ : Calcd.=286.14. Found= $286.47\left[\mathrm{M}^{+}\right]$.

\section{1-[(4-oxoquinazolin-3(4H)-yl)methyl]-3-phenylurea (18c)}

Off white solid, yield $1 \mathrm{~g}(78 \%), \mathrm{mp} 240-242^{\circ} \mathrm{C}$; IR $(\mathrm{KBr})$ v: $3319(\mathrm{NH}), 1677,1613(2 \mathrm{C}=\mathrm{O}) \mathrm{cm}^{-1} ;{ }^{1} \mathrm{H}$ NMR (DMSO- $\left.d_{6}, 500 \mathrm{MHz}\right) \delta: 4.86\left(\mathrm{~s}, 2 \mathrm{H}, \mathrm{CH}_{2}\right), 7.06(\mathrm{~m}, 1 \mathrm{H}, \mathrm{ArH}), 7.31(\mathrm{~m}, 2 \mathrm{H}, \mathrm{ArH}), 7.58(\mathrm{~m}, 3 \mathrm{H}, \mathrm{ArH}), 7.72(\mathrm{~d}, 1 \mathrm{H}$, ArH), $7.85(\mathrm{~m}, 1 \mathrm{H}, \mathrm{ArH}), 8.13(\mathrm{~d}, 1 \mathrm{H}, \mathrm{ArH}), 8.36(\mathrm{~s}, 1 \mathrm{H}, \mathrm{ArH}), 10.45$ (s, 1H, NH) ppm; MS (EI, m/z, $70 \mathrm{eV})$ : Calcd.= 294.11. Found $=294.13\left[\mathrm{M}^{+}\right]$.

\section{1-(4-chlorophenyl)-3-[(4-oxoquinazolin-3(4H)-yl)methyl]urea (18d)}

White solid, yield $1.1 \mathrm{~g}(77 \%), \mathrm{mp} 238-240^{\circ} \mathrm{C}$; IR $(\mathrm{KBr})$ v: $3280(\mathrm{NH}), 1669,1610(2 \mathrm{C}=\mathrm{O}) \mathrm{cm}^{-1} ;{ }^{1} \mathrm{H}$ NMR $\left(\mathrm{DMSO}_{-} \mathrm{d}_{6}, 500 \mathrm{MHz}\right) \delta: 4.85\left(\mathrm{~s}, 2 \mathrm{H}, \mathrm{CH}_{2}\right), 7.38(\mathrm{~m}, 2 \mathrm{H}, \mathrm{ArH}), 7.59(\mathrm{~m}, 3 \mathrm{H}, \mathrm{ArH}), 7.72(\mathrm{~d}, 1 \mathrm{H}, \mathrm{ArH}), 7.85(\mathrm{~m}, 1 \mathrm{H}$, ArH), $8.13(\mathrm{~d}, 1 \mathrm{H}, \mathrm{ArH}), 8.35$ (s, 1H, ArH), 10.59 (s, 1H, NH) ppm; MS (EI, m/z, $70 \mathrm{eV})$ : Calcd.= 328.07. Found= $328.62\left[\mathrm{M}^{+}\right]$.

\section{1-(4-iodophenyl)-3-[(4-oxoquinazolin-3(4H)-yl)methyl]urea (18e)}

gray solid, yield $1.5 \mathrm{~g}(82 \%)$, mp $266-268^{\circ} \mathrm{C}$; IR $(\mathrm{KBr})$ r: $3312(\mathrm{NH}), 1677,1615(2 \mathrm{C}=\mathrm{O}) \mathrm{cm}^{-1}$; ${ }^{1} \mathrm{H}$ NMR (DMSO- $\left.d_{6}, 500 \mathrm{MHz}\right) \delta: 4.84\left(\mathrm{~s}, 2 \mathrm{H}, \mathrm{CH}_{2}\right), 7.42(\mathrm{~m}, 2 \mathrm{H}, \mathrm{ArH}), 7.56(\mathrm{~m}, 1 \mathrm{H}, \mathrm{ArH}), 7.66(\mathrm{~m}, 2 \mathrm{H}, \operatorname{ArH}), 7.72(\mathrm{~m}, 1 \mathrm{H}$, ArH), $7.89(\mathrm{~m}, 1 \mathrm{H}, \operatorname{ArH}), 8.14(\mathrm{~d}, 1 \mathrm{H}, \mathrm{ArH}), 8.35(\mathrm{~s}, 1 \mathrm{H}, \mathrm{ArH}), 10.55(\mathrm{~s}, 1 \mathrm{H}, \mathrm{NH}) \mathrm{ppm}$; MS (EI, $\mathrm{m} / z, 70 \mathrm{eV})$ : Calcd. $=420.01$. Found $=422.26\left[\mathrm{M}^{+}+2\right]$.

\section{1-(2,5-dimethylphenyl)-3-[(4-oxoquinazolin-3(4H)-yl)methyl]urea (18f)}

Gray solid, yield $1.2 \mathrm{~g}(86 \%)$, mp 248-250 ${ }^{\circ} \mathrm{C}$; IR (KBr) v: $3257(\mathrm{NH}), 1662,1608(2 \mathrm{C}=\mathrm{O}) \mathrm{cm}^{-1} ;{ }^{1} \mathrm{H}$ NMR (DMSO-d $6,500 \mathrm{MHz}) \delta: 2.17\left(\mathrm{~s}, 3 \mathrm{H}, \mathrm{CH}_{3}\right), 2.21\left(\mathrm{~s}, 3 \mathrm{H}, \mathrm{CH}_{3}\right), 4.88\left(\mathrm{~s}, 2 \mathrm{H}, \mathrm{CH}_{2}\right), 6.9(\mathrm{~m}, 1 \mathrm{H}, \mathrm{ArH}), 7.09(\mathrm{~m}, 1 \mathrm{H}$, ArH), $7.2(\mathrm{~d}, 1 \mathrm{H}, \operatorname{ArH}), 7.56(\mathrm{~m}, 1 \mathrm{H}, \operatorname{ArH}), 7.71(\mathrm{~d}, 1 \mathrm{H}, \operatorname{ArH}), 7.8(\mathrm{~m}, 1 \mathrm{H}, \mathrm{ArH}), 8.14(\mathrm{~d}, 1 \mathrm{H}, \operatorname{ArH}), 8.36(\mathrm{~s}, 1 \mathrm{H}$, ArH), $9.73(\mathrm{~s}, 1 \mathrm{H}, \mathrm{NH}) \mathrm{ppm} ;{ }^{13} \mathrm{C}$ NMR (DMSO- $\left.66,75 \mathrm{MHz}\right) \delta: 17.39,20.52\left(2 \mathrm{CH}_{3}\right), 48.53\left(\mathrm{CH}_{2}\right), 121.51,125.43$, 126.02, 126.12, 127.09, 127.23, 128.56, 130.19, 134.47, 135.03, 135.50, 148.4, $148.63(\mathrm{ArC}), 160.31,165.57(2 \mathrm{C}=\mathrm{O})$ ppm; MS (EI, $m / z, 70 \mathrm{eV})$ : Calcd.= 322.14. Found $=322.70\left[\mathrm{M}^{+}\right]$.

\section{1-(3-bromophenyl)-3-[(4-oxoquinazolin-3(4H)-yl)methyl]urea (18g)}

Off white solid, yield $1.4 \mathrm{~g}(86 \%)$, mp 244-246 ${ }^{\circ} \mathrm{C}$; IR $(\mathrm{KBr}) v: 3299(\mathrm{NH}), 1673,1610(2 \mathrm{C}=\mathrm{O}) \mathrm{cm}^{-1} ;{ }^{1} \mathrm{H}$ NMR (DMSO- $\left.d_{6}, 500 \mathrm{MHz}\right) \delta: 4.86\left(\mathrm{~s}, 2 \mathrm{H}, \mathrm{CH}_{2}\right), 7.28(\mathrm{~m}, 2 \mathrm{H}, \mathrm{ArH}), 7.5(\mathrm{~m}, 1 \mathrm{H}, \mathrm{ArH}), 7.56(\mathrm{~m}, 1 \mathrm{H}, \mathrm{ArH}), 7.72(\mathrm{~m}, 1 \mathrm{H}$, ArH), $7.85(\mathrm{~m}, 1 \mathrm{H}, \mathrm{ArH}), 7.91(\mathrm{~m}, 1 \mathrm{H}, \mathrm{ArH}), 8.14$ (d, 1H, ArH), 8.35 (s, 1H, ArH), 10.63 (s, 1H, NH) ppm; MS (EI, $m / z, 70 \mathrm{eV})$ : Calcd. $=372.02$. Found $=372.13\left[\mathrm{M}^{+}\right]$.

\section{Methyl [(4-oxoquinazolin-3(4H)-yl)methyl]carbamate (18h)}

To a solution of azide $\mathbf{1 0}(1 \mathrm{~g}, 4.4 \mathrm{mmol})$ in absolute methanol $(10 \mathrm{ml})$ was heated at reflux for $7 \mathrm{~h}$ and the reaction mixture was evaporated under reduced pressure to dryness to give $\mathbf{1 8 h}$ as an off white solid, yield $0.5 \mathrm{~g}(50 \%), \mathrm{mp}$ 172-174 ${ }^{\circ} \mathrm{C}$; IR (KBr) v: $3277(\mathrm{NH}), 1712,1669(2 \mathrm{C}=\mathrm{O}) \mathrm{cm}^{-1} ;{ }^{1} \mathrm{H}$ NMR (DMSO-d $\left.6,500 \mathrm{MHz}\right) \delta: 1.66\left(\mathrm{~s}, 3 \mathrm{H}, \mathrm{CH}_{3}\right)$, $4.36\left(\mathrm{~d}, 2 \mathrm{H}, \mathrm{CH}_{2}\right), 6.72(\mathrm{~m}, 1 \mathrm{H}, \mathrm{ArH}), 6.85(\mathrm{~d}, 1 \mathrm{H}, \mathrm{ArH}), 7(\mathrm{~m}, 1 \mathrm{H}, \operatorname{ArH}), 7.34(\mathrm{~d}, 1 \mathrm{H}, \operatorname{ArH}), 7.54(\mathrm{~s}, 1 \mathrm{H}, \operatorname{ArH}) \mathrm{ppm}$; ${ }^{13} \mathrm{C}$ NMR (DMSO- $\left.d_{6}, 75 \mathrm{MHz}\right) \delta: 47.13\left(\mathrm{CH}_{3}\right), 52.31\left(\mathrm{CH}_{2}\right), 121.61,126.04,127.13,127.20,134.57,147.72,147.84$ (ArC), 157, $159.88(2 \mathrm{C}=\mathrm{O}) \mathrm{ppm}$; MS (EI, $\mathrm{m} / z, 70 \mathrm{eV})$ : Calcd.=233.08. Found $=234.2\left[\mathrm{M}^{+}+1\right]$.

\section{General procedure for the preparation of compounds 18i,j:}

To a solution of azide $\mathbf{1 0}(1 \mathrm{~g}, 4.4 \mathrm{mmol})$ in dry dioxane $(13 \mathrm{~mL})$ the corresponding phenol $(4.4 \mathrm{mmol})$ was added. The reaction mixture was heated at reflux for $10 \mathrm{~h}$, then the solvent was removed under reduced pressure to give a solid.

\section{Phenyl [(4-oxoquinazolin-3(4H)-yl)methyl]carbamate (18i)}

Off white solid, yield $0.62 \mathrm{~g}(48 \%), \mathrm{mp} 212-214{ }^{\circ} \mathrm{C}$; IR (KBr) v: $3377(\mathrm{NH}), 1676,1647(2 \mathrm{C}=\mathrm{O}) \mathrm{cm}^{-1}$; ${ }^{1} \mathrm{H}$ NMR (DMSO- $\left.d_{6}, 500 \mathrm{MHz}\right) \delta: 5.16\left(\mathrm{~s}, 2 \mathrm{H}, \mathrm{CH}_{2}\right), 7.51(\mathrm{~m}, 2 \mathrm{H}, \mathrm{ArH}), 7.66(\mathrm{~m}, 2 \mathrm{H}, \mathrm{ArH}), 7.80(\mathrm{~m}, 2 \mathrm{H}, \mathrm{ArH}), 8.11(\mathrm{~m}, 4 \mathrm{H}$, ArH), 12.26 (brs, $1 \mathrm{H}, \mathrm{NH}$ ) ppm; ${ }^{13} \mathrm{C}$ NMR (DMSO- $\left.d_{6}, 75 \mathrm{MHz}\right) \delta: 52.04\left(\mathrm{CH}_{2}\right), 121.59,122.62,125.82,125.87$, 
$125.91,126.78,126.80,127.12,127.17,127.25,134.35,134.60,145.42,147.79,147.95,148.70,157.54(\operatorname{ArC})$, $160.33,161.03(2 \mathrm{C}=\mathrm{O}) \mathrm{ppm}$.

\section{4-chlorophenyl [(4-oxoquinazolin-3(4H)-yl)methyl]carbamate (18j)}

Off white solid, yield $(0.5 \mathrm{~g}, 35 \%)$, m.p. $214-216{ }^{\circ} \mathrm{C}$. IR $\left(\mathrm{KBr}, \mathrm{cm}^{-1}\right)$ v: $3378(\mathrm{NH}), 1677,1647(2 \mathrm{C}=\mathrm{O}) ;{ }^{1} \mathrm{H}$ NMR $\left(500 \mathrm{MHz}, \mathrm{DMSO}-\mathrm{d}_{6}\right) \delta 5.16\left(\mathrm{~s}, 2 \mathrm{H}, \mathrm{CH}_{2}\right), 7.53(\mathrm{~m}, 2 \mathrm{H}, \mathrm{ArH}), 7.66(\mathrm{~m}, 2 \mathrm{H}, \mathrm{ArH}), 7.79(\mathrm{~m}, 2 \mathrm{H}, \mathrm{ArH}), 8.11(\mathrm{~m}, 3 \mathrm{H}$, $\mathrm{ArH}), 12.26$ (brs, $1 \mathrm{H}, \mathrm{NH}) \mathrm{ppm} ;{ }^{13} \mathrm{C}$ NMR (DMSO- $\left.d_{6}, 75 \mathrm{MHz}\right) \delta: 52.03\left(\mathrm{CH}_{2}\right), 121.59,122.61,125.84,125.91$, $126.78,127.12,127.17,127.24,134.33,134.38,134.60,145.41,147.79,147.95,148.70$ (ArC), 160.33, 161.04 $(2 \mathrm{C}=\mathrm{O}) \mathrm{ppm}$.

\section{[1,2,4]triazolo[4,3-c]quinazoline (20)}

A mixture of $19(1 \mathrm{mmol})$, formic acid/and or triethyl orthoformate $(1 \mathrm{mmol})$ was refluxed for $11 \mathrm{~h}$. The reaction mixture was allowed to cool to room temperature and the formed precipitate was collected by filtration. The precipitate was recrystallized from ethanol to give 20 as yellow powder, yield $0.12 \mathrm{~g}(71 \%), \mathrm{mp} 100-102{ }^{\circ} \mathrm{C} ;{ }^{1} \mathrm{H}$ NMR (DMSO- $\left.d_{6}, 300 \mathrm{MHz}\right) \delta:$ 7.77-7.79 (m, 1H, ArH), 7.84-7.87 (m, 1H, ArH), $7.96(\mathrm{~d}, 1 \mathrm{H}, J=6.5 \mathrm{MHz}, \operatorname{ArH})$, $8.46(\mathrm{~d}, 1 \mathrm{H}, J=6.5 \mathrm{MHz}, \mathrm{ArH}), 9.29(\mathrm{~s}, 1 \mathrm{H}, \mathrm{ArH}), 9.39$ (s, $1 \mathrm{H}, \mathrm{ArH}) \mathrm{ppm} ;{ }^{13} \mathrm{C}$ NMR (DMSO- $\left.d_{6}, 75 \mathrm{MHz}\right) \delta: 116.40$, 122.76, 123.17, 128.45, 129.33, 132.22, 136.96, 139.08, 154.36 (ArC) ppm; MS (EI, $m / z, 70$ eV): Calcd. $=170.06$. Found $=170\left[\mathrm{M}^{+}\right]$; Anal. Calcd. for $\mathrm{C}_{9} \mathrm{H}_{6} \mathrm{~N}_{4}$ (170.18): C, 63.52; H, 3.55; N, 32.92. Found: $\mathrm{C}, 63.49 ; \mathrm{H}, 3.38 ; \mathrm{N}, 32.69$ $\%$.

\section{3-methyl-[1,2,4]triazolo[4,3-c]quinazoline (21).}

A mixture of $19(1 \mathrm{mmol})$, glacial acetic acid $(10 \mathrm{~mL})$, was refluxed for $4 \mathrm{~h}$. The reaction mixture was allowed to cool to room temperature and the formed precipitate was collected by filtration. The precipitate was recrystallized from ethanol to give 21 as pale yellow powder, yield $0.13 \mathrm{~g}(72 \%), \mathrm{mp} 200-202{ }^{\circ} \mathrm{C} ;{ }^{1} \mathrm{H}$ NMR (DMSO$\left.d_{6}, 300 \mathrm{MHz}\right) \delta: 3.03\left(\mathrm{~s}, 3 \mathrm{H}, \mathrm{CH}_{3}\right), 6.71-6.78(\mathrm{~m}, 1 \mathrm{H}, \mathrm{ArH}), 7.83-8.00(\mathrm{~m}, 2 \mathrm{H}, \mathrm{ArH}), 8.03-8.51(\mathrm{~m}, 2 \mathrm{H}, \mathrm{ArH}) \mathrm{ppm}$; ${ }^{13} \mathrm{C}$ NMR (DMSO- $\left.d_{6}, 75 \mathrm{MHz}\right) \delta: 19.59\left(\mathrm{CH}_{3}\right), 116.35,120.56,123.29,123.42,127.83,128.31,129.09,131.30$, $132.61(\mathrm{ArC}) \mathrm{ppm}$; MS (EI, $\mathrm{m} / \mathrm{z}, 70 \mathrm{eV})$ : Calcd.= 184.07. Found $=184\left[\mathrm{M}^{+}\right]$; Anal. Calcd. for $\mathrm{C}_{10} \mathrm{H}_{8} \mathrm{~N}_{4}(184.20)$ : C, $65.21 ; \mathrm{H}, 4.38 ; \mathrm{N}, 30.42$. Found: C, 65.19; H, 4.29; N, $30.29 \%$.

\section{3-phenyl-[1,2,4]triazolo[4,3-c]quinazoline (22)}

A mixture of $19(1 \mathrm{mmol})$, benzoyl chloride $(1 \mathrm{mmol})$ in pyridine $(10 \mathrm{~mL})$, was refluxed for $20 \mathrm{~h}$. The reaction mixture was allowed to cool to room temperature and the formed precipitate was collected by filtration. The precipitate was recystallized from ethanol to give 22 as brown crystals, yield $0.16 \mathrm{~g}(67 \%)$, mp $168-170{ }^{\circ} \mathrm{C} ;{ }^{1} \mathrm{H}$ NMR (DMSO- $\left.d_{6}, 300 \mathrm{MHz}\right) \delta: 7.57(\mathrm{~m}, 3 \mathrm{H}, \mathrm{ArH}), 7.84(\mathrm{~m}, 2 \mathrm{H}, \mathrm{ArH}), 7.94(\mathrm{~m}, 1 \mathrm{H}, \operatorname{ArH}), 8.27(\mathrm{~m}, 2 \mathrm{H}, \operatorname{ArH}), 8.52$ $(\mathrm{m}, 1 \mathrm{H}, \mathrm{ArH}), 9.64(\mathrm{~m}, 1 \mathrm{H}, \mathrm{ArH}) \mathrm{ppm} ;{ }^{13} \mathrm{C}$ NMR (DMSO- $\left.d_{6}, 75 \mathrm{MHz}\right) \delta: 117.51,123.86,127.00,127.40,128.29$, $128.50,129.05,129.73,130.68,131.51,132.27,138.96,142.36,150.73,163.53$ (ArC) ppm; MS (EI, $m / z, 70 \mathrm{eV})$ : Calcd. $=246.09$. Found $=246\left[\mathrm{M}^{+}\right]$; Anal. Calcd. for $\mathrm{C}_{15} \mathrm{H}_{10} \mathrm{~N}_{4}$ (246.27): C, 73.16; H, 4.09; N, 22.75. Found: C, 73.11; $\mathrm{H}, 3.98 ; \mathrm{N}, 22.59 \%$.

\section{General procedure for the synthesis of 23-25:}

To a solution of compound $19(0.16 \mathrm{~g}, 1 \mathrm{mmol})$ in $(20 \mathrm{~mL})$ anhydrous ethanol, ethyl-(ethoxymethylene)cyanoacetate, ethoxymethylene-malonate, and ethoxymethylene-malononitrile, $(1 \mathrm{mmol})$ was added and the reaction mixtures were refluxed for 2-4 h, respectively. The products, which separated on cooling, were collected by filtration and recrystallized from ethanol to give compounds $\mathbf{2 3 - 2 5 .}$

\section{Ethyl 5-amino-1-(quinazolin-4-yl)-1H-pyrazole-4-carboxylate (23)}

Refluxing time: $4 \mathrm{~h}$, yield $0.18 \mathrm{~g}(64 \%), \mathrm{mp} 216-218{ }^{\circ} \mathrm{C}$; $\mathrm{IR}(\mathrm{KBr}) v: 3407,3200\left(\mathrm{NH}_{2}\right) \mathrm{cm}^{-1} ;{ }^{1} \mathrm{H}$ NMR (DMSO- $d_{6}$, $300 \mathrm{MHz}) \delta: 1.3\left(\mathrm{t}, J=6.9 \mathrm{MHz}, 3 \mathrm{H}, \mathrm{CH}_{3}\right), 4.2\left(\mathrm{q}, J=7.5 \mathrm{MHz}, 2 \mathrm{H}, \mathrm{CH}_{2}\right), 6.43-7.25\left(\mathrm{~m}, 6 \mathrm{H}, 4 \mathrm{ArH}\right.$ and $\left.\mathrm{NH}_{2}\right), 7.65$ $(\mathrm{s}, 1 \mathrm{H}$, pyrazole $\mathrm{H}), 9.1(\mathrm{~s}, 1 \mathrm{H}$, pyrimidine $\mathrm{H}) \mathrm{ppm} ;{ }^{13} \mathrm{C}$ NMR (DMSO-d $\left.6,75 \mathrm{MHz}\right) \delta: 12.55\left(\mathrm{CH}_{3}\right), 45.47\left(\mathrm{CH}_{2}\right)$, 102.12, 113.63, 114.23, 117.47, 124.16, 133.22, 134.33, 142.20, 151.16, 155.67, 156.85 (ArC), $176.64(\mathrm{C}=\mathrm{O}) \mathrm{ppm}$; MS (EI, $m / z, 70 \mathrm{eV})$ : Calcd. $=283.11$. Found $=283$ [M+]; Anal. Calcd. for $\mathrm{C}_{14} \mathrm{H}_{13} \mathrm{~N}_{5} \mathrm{O}_{2}(283.11): \mathrm{C}, 59.36 ; \mathrm{H}, 4.63 ; \mathrm{N}$, 24.72. Found: C 59.23, H 4.43, N $24.64 \%$.

\section{Ethyl 5-oxo-1-(quinazolin-4-yl)-4,5-dihydro-1H-pyrazole-4-carboxylate (24)}

Refluxing time: $3 \mathrm{~h}$, yield $0.18 \mathrm{~g}(64 \%)$, mp $189-191{ }^{\circ} \mathrm{C}$; IR $(\mathrm{KBr}) v$ : 1670, $1742(2 \mathrm{C}=\mathrm{O}) \mathrm{cm}^{-1}$; ${ }^{1} \mathrm{H}$ NMR (DMSO$\left.d_{6}, 300 \mathrm{MHz}\right) \delta: 1.06\left(\mathrm{t}, J=7.0 \mathrm{MHz}, 3 \mathrm{H}, \mathrm{CH}_{3}\right), 4.33\left(\mathrm{q}, J=7.5 \mathrm{MHz}, 2 \mathrm{H}, \mathrm{CH}_{2}\right), 5.09(\mathrm{~s}, 1 \mathrm{H}$, pyrazole $\mathrm{H}), 6.80-7.40$ $(\mathrm{m}, 4 \mathrm{H}, 4 \mathrm{ArH}), 7.45(\mathrm{~s}, 1 \mathrm{H}$, pyrazole $\mathrm{H}), 8.76(\mathrm{~s}, 1 \mathrm{H}$, pyrimidine $\mathrm{H}) \mathrm{ppm} ;{ }^{13} \mathrm{C}$ NMR (DMSO- $\left.d_{6}, 75 \mathrm{MHz}\right) \delta: 13.20$ $\left(\mathrm{CH}_{3}\right), 44.45\left(\mathrm{CH}_{2}\right), 112.63,115.23,118.47,124.33,129.22,132.33,146.20,150.55,156.85(\mathrm{ArC}), 166.64,171.12$ 
$(2 \mathrm{C}=\mathrm{O})$ ppm; MS $(\mathrm{EI}, \mathrm{m} / z, 70 \mathrm{eV})$ : Calcd.= 284.09. Found $=284\left[\mathrm{M}^{+}\right]$; Anal. Calcd. for $\mathrm{C}_{14} \mathrm{H}_{12} \mathrm{~N}_{4} \mathrm{O}_{3}(284.09)$ : C, 59.15; H, 4.25; N, 19.71. Found: C, 59.09; H, 4.19; N, $19.61 \%$.

\section{5-Amino-1-(quinazolin-4-yl)-1H-pyrazole-4-carbonitrile (25)}

Refluxing time: $2 \mathrm{~h}$, yield $0.22 \mathrm{~g}(96 \%)$, mp 270-272 ${ }^{\circ} \mathrm{C}$; IR (KBr) v: 3407, $3200\left(\mathrm{NH}_{2}\right), 2209(\mathrm{CN}) \mathrm{cm}^{-1} ;{ }^{1} \mathrm{H}$ NMR (DMSO- $\left.d_{6}, 300 \mathrm{MHz}\right) \delta: 6.41-7.33\left(\mathrm{~m}, 6 \mathrm{H}, 4 \mathrm{ArH}\right.$ and $\left.\mathrm{NH}_{2}\right), 7.60(\mathrm{~s}, 1 \mathrm{H}, \mathrm{ArH}), 9.10(\mathrm{~s}, 1 \mathrm{H}, \mathrm{ArH}) \mathrm{ppm} ;{ }^{13} \mathrm{C}$ NMR $\left(\right.$ DMSO- $\left.d_{6}, 75 \mathrm{MHz}\right) \delta$ : 109.00, 111.67, 112.23, 115.57, 124.75, 129.32, 130.53, 133.75, 142.17, 148.64, 150.13, 154.12 (ArC and CN) ppm; Anal. Calcd. for $\mathrm{C}_{12} \mathrm{H}_{8} \mathrm{~N}_{6}$ (236.08): C, 61.01; H, 3.41; N, 35.58. Found: C, 60.96; H, 3.37; $\mathrm{N}, 35.44 \%$.

\section{4-(3,5-Dimethyl-1H-pyrazol-1-yl)quinazoline (26)}

Refluxing time: $4 \mathrm{~h}$, yield $0.16 \mathrm{~g}(73 \%)$, mp $130-132{ }^{\circ} \mathrm{C} ;{ }^{1} \mathrm{H}$ NMR (DMSO- $\left.d_{6}, 300 \mathrm{MHz}\right) \delta: 1.81\left(\mathrm{~s}, 3 \mathrm{H}, \mathrm{CH}_{3}\right.$ ), $2.42\left(\mathrm{~s}, 3 \mathrm{H}, \mathrm{CH}_{3}\right), 6.01(\mathrm{~s}, 1 \mathrm{H}$, pyrazole $\mathrm{H}), 6.78-7.43(\mathrm{~m}, 4 \mathrm{H}, \mathrm{ArH}), 9.13(\mathrm{~s}, 1 \mathrm{H}$, pyrimidine $\mathrm{H}) \mathrm{ppm} ;{ }^{13} \mathrm{C}$ NMR (DMSO- $\left.d_{6}, 75 \mathrm{MHz}\right) \delta$ : 15.2, $18.5\left(2 \mathrm{CH}_{3}\right), 111.20,114.44,117.20,123.33,127.22,129.33,136.20,140.05,143.85$, , 152.64, $155.12(\mathrm{ArC}) \mathrm{ppm}$; MS (EI, $\mathrm{m} / z, 70 \mathrm{eV})$ : Calcd.= 224.11. Found $=224\left[\mathrm{M}^{+}\right]$; Anal. Calcd. for $\mathrm{C}_{13} \mathrm{H}_{12} \mathrm{~N}_{4}$ (224.11): C, 69.62; H, 5.39; N, 24.98. Found: C 69.47; H 5.29; N, $24.71 \%$.

\section{3-Methyl-1-(quinazolin-4-yl)-1H-pyrazol-5(4H)-one (27)}

To a solution of compound $19(0.16 \mathrm{~g}, 1 \mathrm{mmol})$ in glacial acetic acid $(20 \mathrm{~mL})$, ethyl acetoacetate $(1 \mathrm{mmol})$ was added and the reaction mixture was refluxed for $6 \mathrm{~h}$. The solvent was then removed under reduced pressure and the obtained product was recrystallized from ethanol to give compound 27. Yield $0.16 \mathrm{~g}(73 \%), \mathrm{mp} 140-142{ }^{\circ} \mathrm{C} ; \mathrm{IR}(\mathrm{KBr})$ $v: 1690(\mathrm{C}=\mathrm{O}) \mathrm{cm}^{-1},{ }^{1} \mathrm{H}$ NMR (DMSO-d6, $\left.300 \mathrm{MHz}\right) \delta: 2.73\left(\mathrm{~s}, 3 \mathrm{H}, \mathrm{CH}_{3}\right), 3.89\left(\mathrm{~s}, 2 \mathrm{H}, \mathrm{CH}_{2}\right), 7.22-8.01(\mathrm{~m}, 4 \mathrm{H}$, ArH), $9.45(\mathrm{~s}, 1 \mathrm{H}$, pyrimidine $\mathrm{H}) \mathrm{ppm} ;{ }^{13} \mathrm{C}$ NMR (DMSO-d6, $\left.75 \mathrm{MHz}\right) \delta: 19.25\left(\mathrm{CH}_{3}\right), 35.23\left(\mathrm{CH}_{2}\right), 114.57,123.75$, 128.32, 131.53, 131.85, 133.15, 141.05, 148.64, $160.12(\mathrm{ArC}), 166.23(\mathrm{C}=\mathrm{O}) \mathrm{ppm}$; MS $(\mathrm{EI}, \mathrm{m} / \mathrm{z}, 70 \mathrm{eV})$ : Calcd.= 226.09. Found $=226\left[\mathrm{M}^{+}\right]$; Anal. Calcd. for $\mathrm{C}_{12} \mathrm{H}_{10} \mathrm{~N}_{4} \mathrm{O}$ (226.09): C, 63.71; H, 4.46; N, 24.76. Found: C, 63.56; H, $4.49 ; \mathrm{N}, 24.64 \%$.

General procedure for the preparation of compounds $29 a-c$ :

To a suspension of 4-hydrazinylquinazoline $19(0.16 \mathrm{~g}, 1 \mathrm{mmol})$ in dioxane $(50 \mathrm{~mL})$ was added acetic acid $(0.1 \mathrm{ml})$ and the appropriate $5(4 \mathrm{H})$-oxazolone $(\mathbf{2 8 a}-\mathbf{c})(1 \mathrm{mmol})$. The mixture was refluxed for an appropriate time $(36-72 \mathrm{~h})$ and then the mixture was poured into cold water $(100 \mathrm{ml})$. The precipitate was filtered and recrystallized from ethyl acetate to give the corresponding imidazoloquinazolinone derivatives 29a-c as yellow crystals.

\section{4-Benzylidene-2-phenyl-1-(quinazolin-4-ylamino)-1H-imidazol-5(4H)-one (29a)}

Reflux time: $48 \mathrm{~h}$, yield $0.28 \mathrm{~g}(71 \%)$, mp 335-337 ${ }^{\circ} \mathrm{C}$ (decomp.); IR (KBr) v: $3408(\mathrm{NH}), 1682$ (C=O), 1617, 1602 $(2 \mathrm{C}=\mathrm{N}) \mathrm{cm}^{-1} ;{ }^{1} \mathrm{H}-\mathrm{NMR}$ (DMSO- $\left.d_{6}, 300 \mathrm{MHz}\right) \delta: 7.19-8.40\left(\mathrm{~m}, 16 \mathrm{H}\right.$, vinyl, ArH), $10.97(\mathrm{~s}, 1 \mathrm{H}, \mathrm{NH}) \mathrm{ppm} ;{ }^{13} \mathrm{C}-\mathrm{NMR}$ $\left(\mathrm{DMSO}-d_{6}, 75 \mathrm{MHz}\right) \delta: 123.54,126.12,127.20,128.21,128.50,128.63,128.86,130.01,130.31$ 130.90, 132.70, 132.08, 135.44, 144.22, 149.62, 158.41, $163.12(\mathrm{ArC}), 170.73(\mathrm{C}=\mathrm{O}) \mathrm{ppm}$; MS (EI, $\mathrm{m} / \mathrm{z}, 70 \mathrm{eV})$ : Calcd $=391.14$. Found $=391\left[\mathrm{M}^{+}\right]$; Anal. Calcd. For $\mathrm{C}_{24} \mathrm{H}_{17} \mathrm{~N}_{5} \mathrm{O}$ (391.14): C, 73.64; H, 4.38; N, 17.89. Found: C, 73.42; H, 4.29; N, $17.73 \%$.

\section{4-(4-Chlorobenzylidene)-2-phenyl-1-(quinazolin-4-ylamino)-1H-imidazol-5(4H)-one (29b)}

Reflux time: $72 \mathrm{~h}$, yield $0.18 \mathrm{~g}(43 \%)$, mp 352-354 ${ }^{\circ} \mathrm{C}$ (decomp.); IR (KBr) v: 3394 (NH), 1692 (C=O), 1626, 1602 $(2 \mathrm{C}=\mathrm{N}) \mathrm{cm}^{-1} ;{ }^{1} \mathrm{H}-\mathrm{NMR}$ (DMSO- $\left.d_{6}, 300 \mathrm{MHz}\right) \delta: 7.02-8.33\left(\mathrm{~m}, 15 \mathrm{H}\right.$, Vinyl, ArH), $10.98(\mathrm{~s}, 1 \mathrm{H}, \mathrm{NH}) \mathrm{ppm} ;{ }^{13} \mathrm{C}-\mathrm{NMR}$ $\left(\mathrm{DMSO}-d_{6}, 75 \mathrm{MHz}\right) \delta: 125.22,126.78,127.66,128.26,128.80,128.93,129.86,131.01,131.31$ 131.90, 133.70, 134.18, 136.13, 145.25, 150.64, 159.12, $164.15(\mathrm{ArC}), 172.09(\mathrm{C}=\mathrm{O}) \mathrm{ppm}$; MS (EI, m/z, $70 \mathrm{eV}):$ Calcd. $=435.10$. Found $=435\left[\mathrm{M}^{+}\right]$; Anal. Calcd. For $\mathrm{C}_{24} \mathrm{H}_{16} \mathrm{ClN}_{5} \mathrm{O}$ (425.10): C, 67.69; H, 3.79; N, 16.44. Found: C, 67.42; H, 3.59; N, $16.32 \%$.

4-(4-Methoxybenzylidene)-2-phenyl-1-(quinazolin-4-ylamino)-1H-imidazol-5(4H)-one (29c)

Reflux time: $24 \mathrm{~h}$, yield $0.35 \mathrm{~g}(83 \%)$, mp 356-358 ${ }^{\circ} \mathrm{C}$ (decomp.); IR (KBr) v: $3390(\mathrm{NH}), 1692(\mathrm{C}=\mathrm{O}), 1632,1606$ $(2 \mathrm{C}=\mathrm{N}) \mathrm{cm}^{1}{ }^{1} ;{ }^{1} \mathrm{H}-\mathrm{NMR}\left(\mathrm{DMSO}-d_{6}, 300 \mathrm{MHz}\right) \delta: 3.85\left(\mathrm{~s}, 3 \mathrm{H}, \mathrm{OCH}_{3}\right), 7.12-8.38(\mathrm{~m}, 15 \mathrm{H}$, vinyl, ArH), $10.91(\mathrm{~s}, 1 \mathrm{H}$, $\mathrm{NH}) \mathrm{ppm} ;{ }^{13} \mathrm{C}-\mathrm{NMR}\left(\mathrm{DMSO}-d_{6}, 75 \mathrm{MHz}\right) \delta: 55.23\left(\mathrm{CH}_{3}\right), 124.15,125.78,126.33,127.25,127.60,128.03,128.36$, 129.51, 129.55, 130.45, 132.56, 134.66, 136.78, 146.77, 152.64, 156.14, 164.77 (ArC), 165.79 (C=O) ppm; MS (EI, $\mathrm{m} / z, 70 \mathrm{eV})$ : Calcd. $=421.15$. Found $=421\left[\mathrm{M}^{+}\right]$; Anal. Calcd. For $\mathrm{C}_{25} \mathrm{H}_{19} \mathrm{~N}_{5} \mathrm{O}_{2}(421.15): \mathrm{C}, 71.25 ; \mathrm{H}, 4.54 ; \mathrm{N}, 16.62$. Found: C, $71.12 ; \mathrm{H}, 4.41 ; \mathrm{N}, 16.42$.

\section{Results and disscussions}


The synthetic strategies and the biological evaluations on the pyrido[1,2-c] quinazolinone derivatives are limited, only two synthetic approach were reported. The first one included the synthesis from ethyl 2-(quinazolone)-5-oxo-2,5dihydroisoxazole-4-carboxylates as starting materials, in which reaction first with $\mathrm{NaN}_{3}$ followed by base-catalyzed rearrangement [28]. The second method based on the ring closure of the 2-o-amino-phenyl-6-phenylpyrimidine4(3H)one with formic acid [29]. Both producers are long synthetic way and limited to synthesize various derivatives. Since many analogues of this ring system showed marked biological activity [30], we have investigated the synthesis of this scaffold, and herein we present our results.

Quinazolin-4-ol (1) was prepared as described in the literature, [31] Upon refluxing with freshly distilled thionyl chloride in presence of catalytic amount of DMF, compound 1 yielded the corresponding 4-chloro derivative 2 [32]. Which followed by its reaction with sodium azide in DMF to give 4-azidoquinazoline $(\mathbf{3})$ in $71 \%$ yield. Treating of compound 3 with triphenylphosphine gave phosphrane derivative 4 in $69 \%$ yield. On hydrolysis of the latter compound 4 by glacial acetic acid gave quinazolin-4-amine (5) in $76 \%$ yield. The interaction of aminoquinazoline 5 with malonate derivatives afforded pyrimidoqunazoline derivatives 6a-d in 67-92\% yields (Scheme 1). The structures of compounds $\mathbf{3}, \mathbf{4}$ and $\mathbf{5}$ were established based on elemental analysis and spectral data.

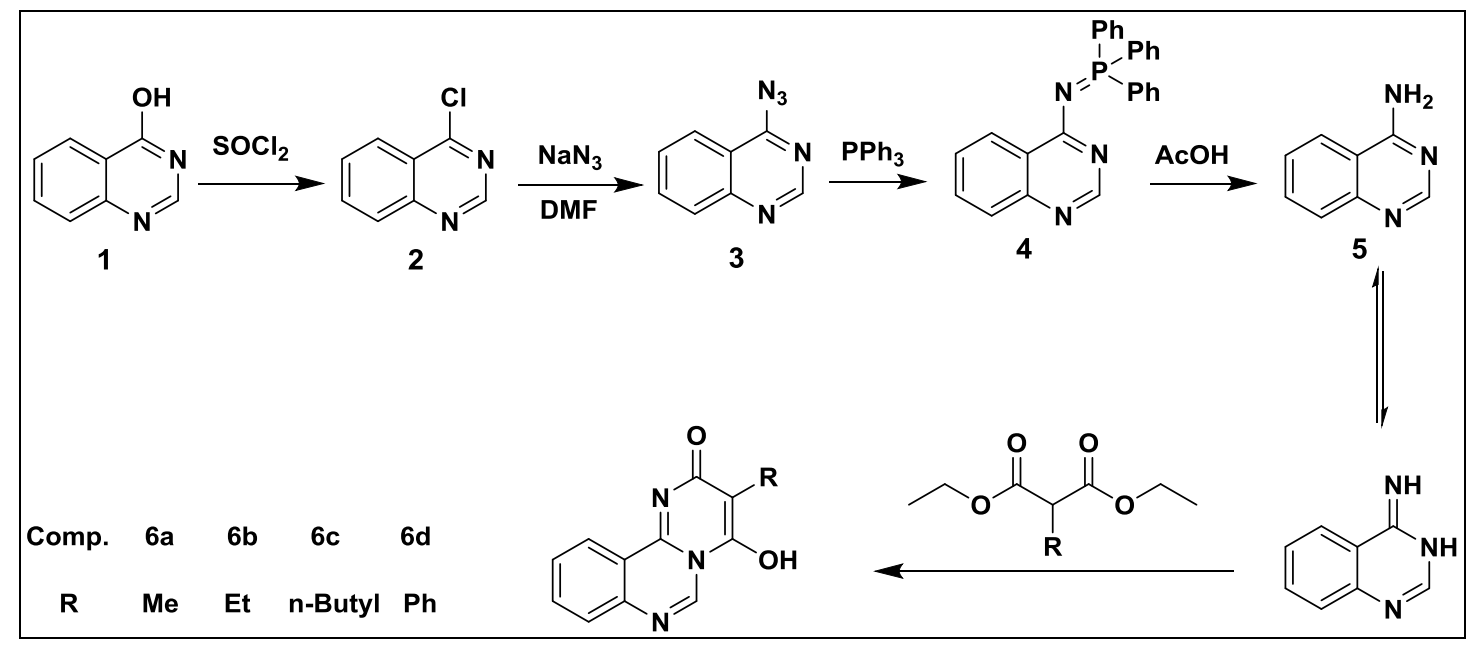

Scheme 1. Fomation of alkyl-pyrido[1,2-c]quinazolinone derivatives

Quinazolone derivatives bearing different moieties for example hydrazide, ester, or imine derivatives at the N-3 position are well known to exhibit powerful antimicrobial activities [33-35]. In this context, our main target was dedicated at the design and the synthesis of novel series of N-3 substituted quinazolone derivatives according to the synthetic methods showed in (Scheme 2). The starting material ethyl 2-(4-oxoquinazolin-3(4H)-yl)acetate (8) was synthesized via procedure as described in our earlier report [26]. Hydrazinolysis of (8) at room temperature afforded 2-(4-oxoquinazolin-3(4H)-yl)acetohydrazide (9) followed by the treatment with $\mathrm{NaNO}_{2}$ in the presence of $\mathrm{HCl}$ or acetic acid to form 2-(4-oxoquinazolin-3(4H)-yl)acetyl azide (10). When the hydrazinolysis was performed under reflux conditions, afforded the key intermediate 3-aminoquinazolin-4(3H)-one (11). Treatment of this intermediate with the appropriate aldehyde in the presence of acetic acid gave the corresponding-desired imine derivatives (13a-f). On the other hand, the reaction of $\mathbf{1 1}$ with acetyl bromide or benzoyl chloride lead to the formation of $\mathrm{N}$-(4oxoquinazolin-3(4H)-yl)acetamide (14a) or $N$-(4-oxoquinazolin-3(4H)-yl)benzamide (14b), respectively.

Construction of the final products 17a-c can be done by reaction between 2-chloro- $N$-(4-oxoquinazolin-3(4H)yl)acetamide (16) and the commercially available secondary amine or the aniline derivatives in the presence of potassium carbonate as a base in acetonitrile and stirring at room temperature for $24 \mathrm{~h}$ to afford the final products in $81 \%$ to $83 \%$ yield as showed in (Scheme 3 ).

The synthetic route used to prepare carbamides 18a-g and carbamates derivatives $\mathbf{1 8 h}-\mathbf{j}$ is outlined in (Scheme 4). In which a Curtius rearrangement of the acyl azide $\mathbf{1 0}$ was carried out by refluxing in dioxane. In details, refluxing the acyl azide $\mathbf{1 0}$ with secondary amine or aniline derivatives gave the corresponding carbamides $\mathbf{1 8 a}$ to $\mathbf{1 8 g}$, while the refluxing with methanol or phenol derivatives gave the corresponding carbamates $\mathbf{1 8 h}$ to $\mathbf{1 8 j}$ (Scheme 4). 


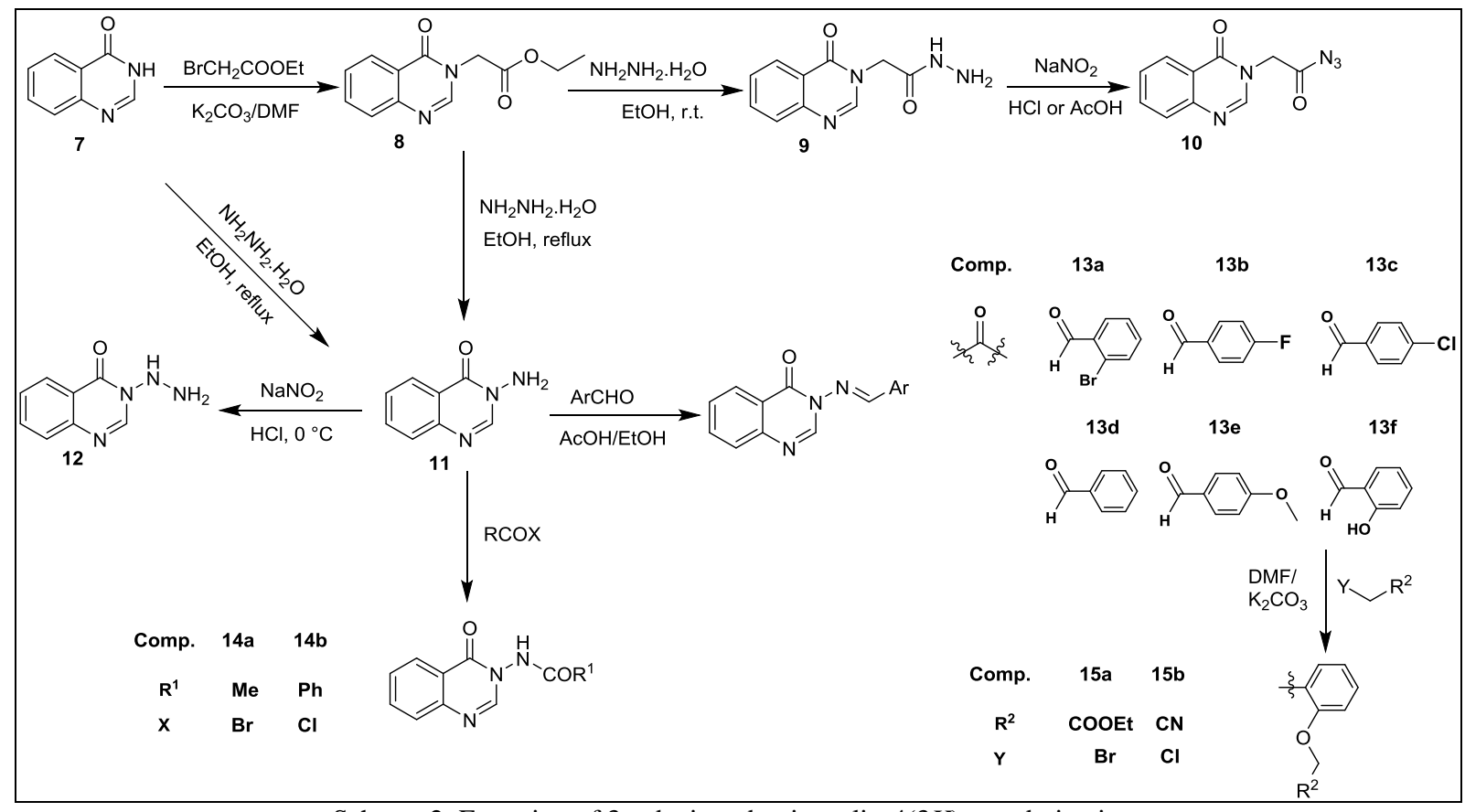

Scheme 2. Fomation of 3-substituted quinazolin-4(3H)-one derivatives

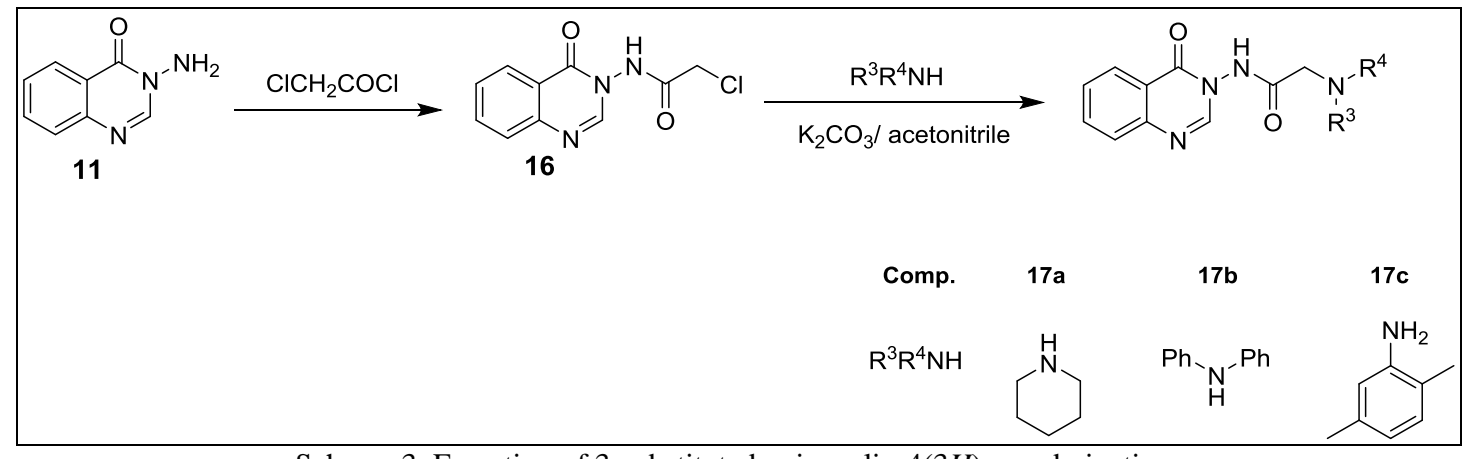

Scheme 3. Fomation of 3-substituted quinazolin-4(3H)-one derivatives

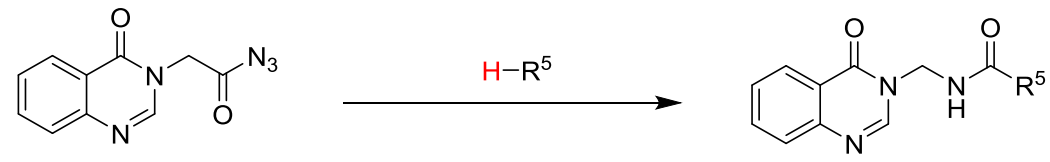

10

$18 \mathrm{a}-\mathrm{\jmath}$

(2) Product




(18)

Scheme 4. Fomation of 3- carbamides and carbamates quinazolin-4(3H)-one derivatives

The construction of 5-substituted or not substituted 1,2,4-triazole moiety fused from the 3,4 side with quinazoline heterocyclic ring from the $c$ side leads to 5-substituted or not substituted 1,2,4-triazolo[4,3-c]quinazoline having various biological activities. Different derivatives from 1,2,4-triazolo[4,3-c] quinazoline have been synthesized as follow, the starting material quinazoline-4-thiol produced 4-hydrazinylquinazoline (19) in moderate yield when subjected to condensation with hydrazine hydrate in alcoholic medium [36]. The key intermediate 4-hydrazinyl derivative 19 was readily converted to the fused triazolo derivatives 20-22 (Scheme 5) by reaction with different reagents, triethylorthoformate, acetic acid, and/or benzoyl chloride to give the triazoloquinazoline derivatives $\mathbf{2 0 - 2 2}$ in moderate yield $67-72 \%$. The structures of all the newly synthesized compounds $\mathbf{2 0 - 2 2}$ were confirmed by ${ }^{1} \mathrm{H}$ NMR, ${ }^{13} \mathrm{C}$ NMR and element analysis data, explained in the experimental part.

In order to prepared the spyropyrazolo quinazoline derivatives, 4-hydrazinylquinazoline (19) was dissolved in ethanol and refluxed with ethyl-(ethoxymethylene)-cyanoacetate, ethoxymethylene-malonate, ethoxymethylenemalononitrile, acetyl acetone and ethylacetoacetate (in $\mathrm{AcOH}$ ) afforded the corresponding substituted pyrazole derivatives 23-27, respectively (Scheme 5). The structures of the latter compounds were confirmed on the basis of their elemental analysis and spectral data (cf. Experimental). The IR spectra of compounds 23 and 25 showed absorption bands characteristic for $\mathrm{NH}_{2}$ and $\mathrm{C} \equiv \mathrm{N}$ groups, while those of compound $\mathbf{2 4}$ and $\mathbf{2 7}$ revealed absorption bands characteristic for $\mathrm{C}=\mathrm{O}$ (ester) and $\mathrm{C}=\mathrm{O}$ (keton). Also, the ${ }^{1} \mathrm{H}$ NMR spectra showed signals at $\delta=6.41-7.33$ ppm due to $\mathrm{NH}_{2}$ for compounds 23 and 25 respectively.

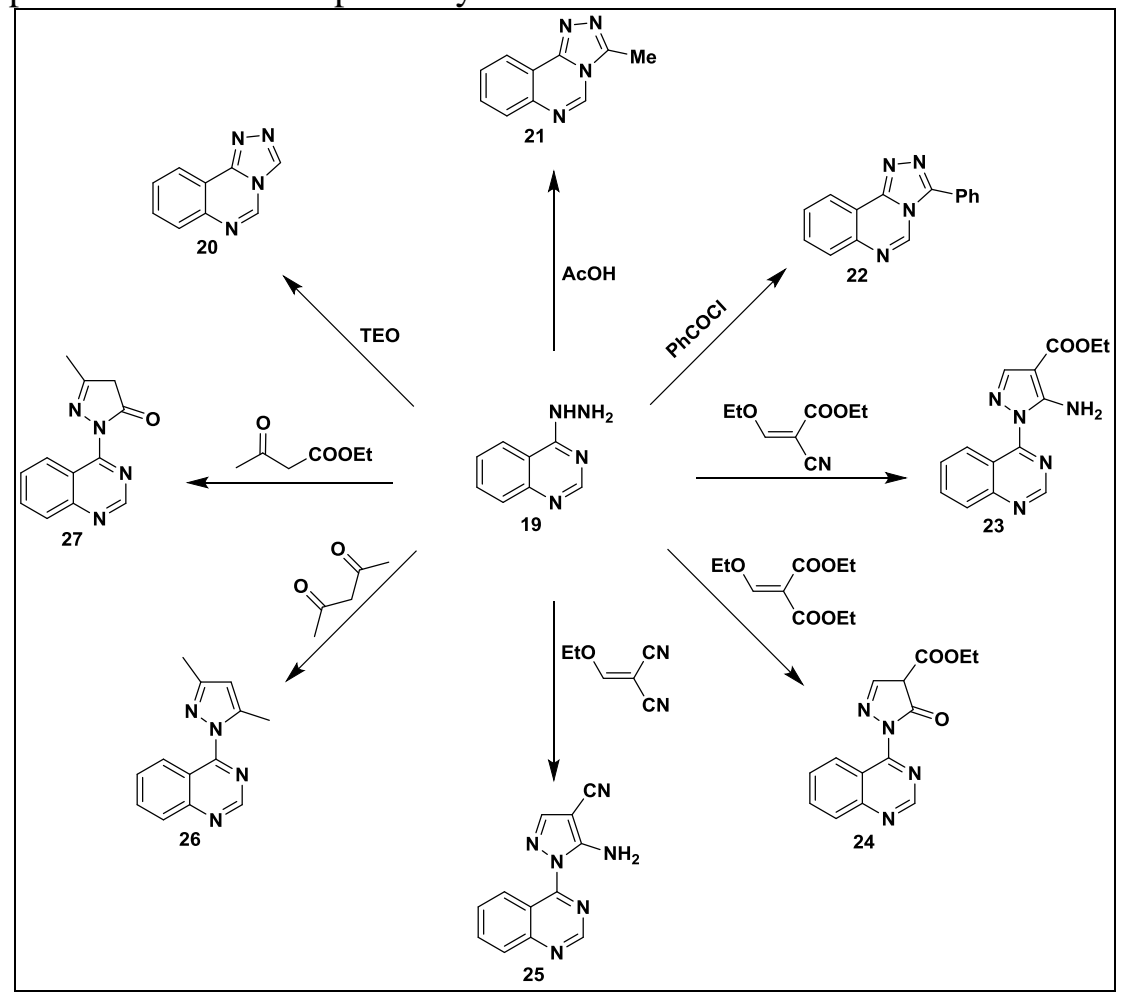

Scheme 5. Fomation of triazoloquinazoline and spyropyrazolo quinazoline derivatives

2-Phenyl-5(4H)-oxazolone derivatives (28a-c) were prepared separately from hippuric acid, acetic anhydride, sodium acetate and an appropriate aldehyde or ketone [37]. In the last step 4-hydrazinylquinazoline (19) was reacted with the 2-phenyl-5(4H)-oxazolone derivatives (28a-c) in the presence of acetic acid (Scheme 6). In this reaction, 4hydrazinylquinazoline acts as a nucleophile. Compound (19) attacks the carbonyl group of the oxazolone ring and the ring is cleaved, then the 4-imidazolin-5-one ring is formed. It was expected to obtain compound (30) but instead compound (29a-c) were formed (reaction mechanism in Scheme 6). This reaction cannot be carried out with aldehydes or ketones containing electron-withdrawing substituents such as 4- fluorobenzaldehyde and 2,2,2-trifluoro-1phenylethanone or basic substituents such as $N, N$-dimethylbenzaldehyde. Basic groups react with acetic acid and convert it to an electron-withdrawing group. Electron-withdrawing substituents prevent the formation of the 2hydroxy-imidazolidinyl ring (29) (Scheme 6). 
<smiles>[R]C([R])=C1N=C(c2ccccc2)N(Nc2ncnc3ccccc23)C1=O</smiles>

$\begin{array}{cccc}\text { Comp. } & 29 a & 29 b & 29 c \\ \mathrm{R}^{1} & \mathrm{C}_{6} \mathrm{H}_{5} & 4-\mathrm{Cl}-\mathrm{C}_{6} \mathrm{H}_{4} & 4-\mathrm{OCH}_{3}-\mathrm{C}_{6} \mathrm{H}_{4} \\ \mathrm{R}^{2} & \mathrm{H} & \mathrm{H} & \mathrm{H}\end{array}$

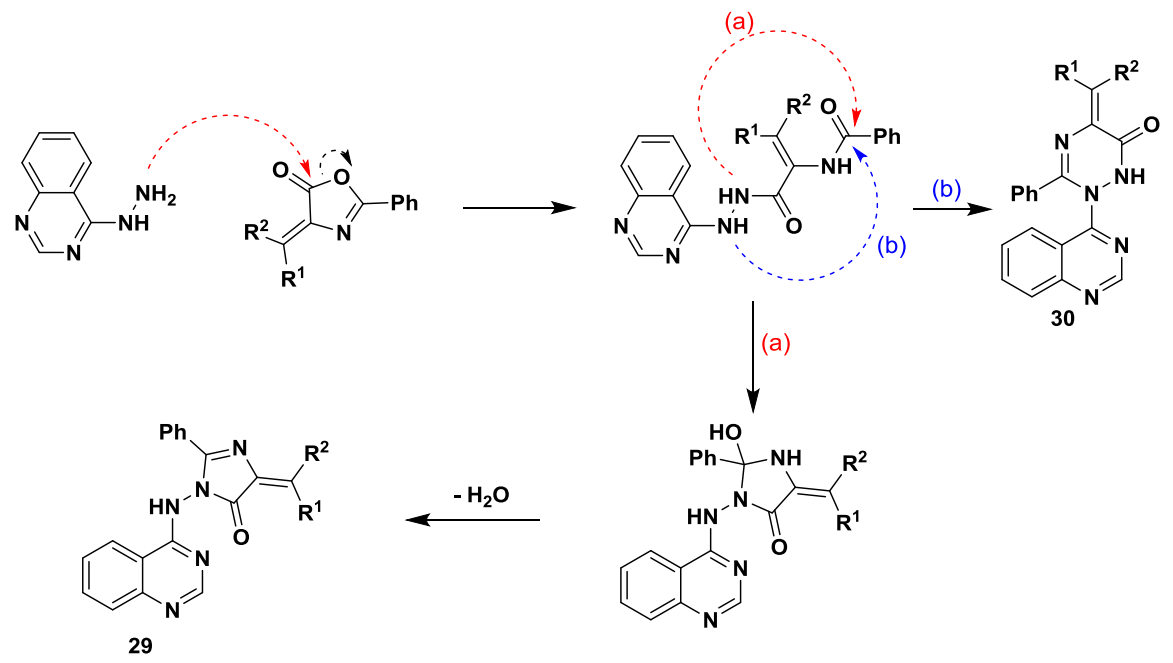

Scheme 6. Formation of Imidazoline derivatives

\section{Antimicrobial activity}

The antimicrobial activities of the 3-, 4-substituted, and 3,4-di substituted quinazoline derivatives were evaluated by the agar diffusion method under the regulations made by Clinical and Laboratory Standards Institute (CLSI) [38]. The inhibition zone for each derivative measured by ruler to determine its size (in $\mathrm{mm}$ ) and compared with the inhibition zone produced by the standard drugs. The quinazoline derivatives were evaluated for antimicrobial activity against bacteria (Gram-positive bacteria: Bacillus subtilis and Staphylococcus aureus; Gram-negative bacteria: Escherichia coli and Proteus vulgaris), and fungi (Candida albicans and Aspergillus flavus). Gentamycin used as standard drug for the bacterial strains, while Ketoconazole was used as standard drug for the fungi strains (table 1).

The antimicrobial evaluation of the synthesized derivatives displayed a moderate activity of some compounds in comparison with the reference drugs, for example compounds 13d, 15a, 17b, 18b, 18d, 25 and 29a-c. Among the novel derivatives, the pyrimidoqunazoline derivative $\mathbf{6 c}$ elicited the most active compound against all the tested strains.

Table 1

ANTIMICROBIAL ACTIVITY OF SYNTHESIZED COMPOUNDS

\begin{tabular}{|c|c|c|c|c|c|c|}
\hline \multirow[t]{2}{*}{ Compound } & \multicolumn{2}{|c|}{ Gram Positive } & \multicolumn{2}{|c|}{ Negative Gram } & \multicolumn{2}{|c|}{ Fungi } \\
\hline & $\begin{array}{c}\text { Bacillus } \\
\text { subtilis }\end{array}$ & $\begin{array}{c}\text { Staphylococcus } \\
\text { aureus }\end{array}$ & E Coli & $\begin{array}{l}\text { Proteus } \\
\text { vulgaris }\end{array}$ & $\begin{array}{l}\text { Candida } \\
\text { albicans }\end{array}$ & $\begin{array}{l}\text { Aspergillus } \\
\text { flavus }\end{array}$ \\
\hline \multirow[t]{2}{*}{ Control } & \multicolumn{2}{|c|}{ Gentamycin } & \multicolumn{2}{|c|}{ Gentamycin } & \multicolumn{2}{|c|}{ Ketoconazole } \\
\hline & 26 & 24 & 30 & 25 & 20 & 16 \\
\hline $6 \mathbf{a}$ & - & - & 8 & - & - & - \\
\hline $6 c$ & 24 & 18 & 23 & 22 & 28 & 25 \\
\hline $6 d$ & - & - & - & - & - & - \\
\hline 13a & - & - & - & - & - & - \\
\hline 13b & - & - & - & 15 & - & - \\
\hline $13 \mathrm{c}$ & - & - & - & 12 & - & - \\
\hline 13d & - & - & 9 & 10 & - & - \\
\hline $13 \mathrm{e}$ & - & - & - & 14 & - & - \\
\hline 13f & - & - & - & 16 & - & - \\
\hline $14 \mathbf{a}$ & - & - & - & - & - & - \\
\hline $14 \mathrm{~b}$ & - & - & - & - & - & - \\
\hline
\end{tabular}




\begin{tabular}{|c|c|c|c|c|c|c|}
\hline $15 a$ & - & - & 12 & 10 & - & - \\
\hline $15 \mathrm{~b}$ & - & - & - & - & - & - \\
\hline $17 a$ & - & - & - & 12 & - & - \\
\hline $17 b$ & 8 & - & - & - & 10 & - \\
\hline $17 \mathrm{c}$ & - & - & - & 8 & - & - \\
\hline $18 \mathbf{a}$ & - & - & - & - & - & - \\
\hline $18 b$ & - & 9 & - & - & 8 & - \\
\hline $18 c$ & - & - & - & 10 & - & - \\
\hline 18d & - & - & - & - & 9 & - \\
\hline $18 \mathrm{e}$ & - & - & - & - & - & - \\
\hline 18f & - & - & - & - & - & - \\
\hline $18 \mathrm{~g}$ & - & - & - & - & - & - \\
\hline $18 i$ & - & - & - & 8 & - & - \\
\hline $\mathbf{1 8 j}$ & - & - & - & - & - & - \\
\hline 21 & - & - & - & - & - & - \\
\hline 23 & 9 & - & 8 & - & - & - \\
\hline 25 & 11 & 12 & 10 & - & - & - \\
\hline 22 & - & - & - & - & - & - \\
\hline 24 & - & - & - & - & - & - \\
\hline $29 a$ & 15 & 11 & 15 & 13 & - & - \\
\hline $29 b$ & 8 & 9 & 8 & - & - & - \\
\hline $29 c$ & 11 & 10 & - & - & - & - \\
\hline 26 & - & - & - & - & - & - \\
\hline 27 & - & - & - & - & - & - \\
\hline
\end{tabular}

\section{Conclusions}

In conclusion, a novel series of 3-, 4-substituted, and 3,4-di substituted quinazoline derivatives were designed and synthesized. The structure of the novel derivatives have been elucidated using different spectroscopic techniques (IR, NMR spectra and EI-MS). The antimicrobial activities of the most novel quinazoline derivatives have been evaluated against Gram-positive, Gram-negative bacteria, and fungi. The most active compound against all the tested strains was recorded for compound $\mathbf{6 c}$. whereas compounds 13d, 15a, 17b, 18b, 18d, 25 and 29a-c of the newly synthesized compounds exhibited moderate antimicrobial activities. So that, the pyrimidoqunazoline derivative $\mathbf{6 c}$ could be useful as a hit scaffold for further modifications to synthesized more active derivatives.

Acknowledgments: This project was supported by the Deanship Research at Prince Sattam bin Abdulaziz University and Menoufia University, Chemistry Department.

\section{References}

1. VITAKU, E., SMITH, D.T., NJARDARSON, J.T., J. Med. Chem., 57, 2014, p. 10257

2. SURESHA, G.P., PRAKASHA, K.C., SHIVAKUMARA, K.N., KAPFO, W., GOWDA, D.C., Int. J. Pept. Ther., 15, 2009 , p. 25

3. ALAGARSAMY, V., RAJA, V.S., VANIKAVITHA, G., PALUCHAMY, V., AMUTHALAKSHMI, S., CHANDRAN, M.R., SUJIN, A.A., THANGATHIRUATHY, A., REVATHI, R., Biol. Pharm. Bull., 25, 2002, p. 1432

4. ALAGARSAMY, V., THANGATHIRUATHY, A., MANDAL, S.C., RAJASEKARAN, S., VIJAYAKUMAR, S., REVATHI, R., ANBURAJ, J., ARUNKUMAE, S., RAJESH, S., Ind. J. Pharm. Sci., 68, 2006, p. 108

5. MURGAN, V., THOMAS, C.C., RAMA, G.V.S., KUMAR, E.P., Ind. J. Pharm. Sci., 65, 2003, p. 386

6. NANDY, P., VISHALAKSHI, M.T., BHAT, A.R., Ind. J. Heterocycl. Chem., 15, 2006, p. 293

7. HORTON, D.A., BOURNE, G.T., SMYTHE, M.L., Chem. Rev., 103, 2003, p. 893

8. MICHAEL, J.P., Nat. Prod. Rep., 21, 2004, p. 650

9. RAW, V.J., SRIMAL, R.C., KUSHWAHA, D.S., MISHRA, L., J. Prakt. Chem., 323, 1990, p. 629

10. BEKHIT, A.A., HBBIB, N.S., El-BEKHIT, A., Boll. Chem. Farm., 140, 2001, p. 297

11. JIANG, J.B., HESSON, D.P., DUSAK, B.A., DEXTER, D.1., KANG, G.T., J. Med. Chem., 33, 1990, p. 1721

12. LOPEZ, E.S., ROSALES, M.E., CANELON, E.C., VALVERODE, A.E., NARVAEZ, R.C., CHARRIES, J.E., GIANNINI, F.A., ENRIZ,

R.D., CARRASCO, M., ZACCHINO, S., Heterocycl. Cammun., 7, 2000, p. 473

13. FARGHALY, A.O., MOHARRAM, A.M., Boll-Chim. Farm., 138, 1999, p. 280

14. GHORAB, M.M., El-SHARIEF, A.M.Sh., AMMAR, Y.A., MOHAMED, Sh.I., Phosphorous. Sulfur and Silicon., 173, 2001, p. 223

15. HARDTMANN, G.E., KATHAWALA, F.G.U.S., Pat 4,053,600, 1977 (Chem. Abstr., 88, 1978, p. 22970)

16. ZHANG, L.-X., ZHANG, A.-J., CHEN, X.-X., LEI, X.-X., NAN, X.-Y., CHEN, D.-Y., ZHANG, Z.-Y., Molecules., 7, 2002 , p. 681

17. ABDOU, M.I., SALEH, A.M., ZOHDI, H.F., Molecules., 9, 2004, p. 109

18. Al-SAADI, M.S., ROSTOM, S.A.F., FAIDALLAH, H.M., Arch. Pharm. Chem. Life Sci., 341, 2008, p. 181

19. CAPDEVILLE, R., BUCHDUNGER, E., ZIMMERMANN, J., MATTER, A., Nat. Rev. Drug Disc., 1, 2002, p. 493

20. GADHACHANDA, V.R., WU, B., WANG, Z., KUHEN, K.L., CALDWELL, J., ZONDLER, H., WALTER, H., HAVENHAND, M., HE,

Y., Bioorg. Med. Chem. Lett., 17, 2007, p. 260

21. KUCUKGUZEL, S.G., ROLLAS, S., ERDENIZ, H., KIRAZ, M., EKINCI, A.C., VIDIN, A., Eur. J. Med. Chem., 35, 2000 , p. 761

22. GENIN, M.J., BILES, C., KEISER, B.J., POPPE, S.M., SWANEY, S.M., TARPELY, W.G., YAGI, Y., ROMERO, D.L., J. Med. Chem., 43, 2000, p. 1034

23. DAidone, G., MAGgio, B., Plescia, S., RAFFA, D., MUSIU, C., MiLIA, C., PERRA, G., MARONGIU, M.E., Eur. J. Med. Chem., 33, 1998, p. 375

24. El-ESSAWY, F.A., J. Heterocycl. Chem., 47, 2010, p. 323

25. El-ESSAWY, F.A., Synth. Communi., 40, 2010, p. 877 
26. BOSHTA, N.M., El-ESSAWY, F.A., AMMAR, R.M., ISMAIL, A.E.-H., WAHBA, N.E., Monatsh. Chem., 147, 2016, p. 2031

27. El-ESSAWY, F.A., BOSHTA, N.M., ALOTAIBI, M.A., ELSAYED, M.S., TARABEESM, R., SALEH, E.A., Res. Chem. Intermed., 42, 2016, p. 8157

28. ANG, K.H., DONATI, C., DONKOR, A., PRAGER, R.H., Aust. J. Chem., 45, 1992, p. 2037

29. PLESCIA, S., AJELLO, E., SPRIO, V., MARINO, M.L., J. Heterocycl. Chem., 11, 1974, p. 603

30. WANG, Y., LIU, J.-Q., WANG, X.-S., J. Heterocycl. Chem., 54, 2017, p. 3440

31. OSWALD, S.T., GHANSHYAM, V.P., J. Org. Chem., 41, 1976, p. 838

32. SCHOENOWSKY, H., SACHSE, B.Z., NATURFORSH, B., Anorg. Chem. Org. Chem., 37, 1982, p. 907

33. YAN, B.-R., LV, X.-Y., DU, H., GAO, M.-N., HUANG, J., BAO, X.-P., Chemical. Papers, 70, 2016, p. 983

34. KOVALENKO, S.I., Farmatsevtichnii. Zhurnal. (Kiev)., 1, 1999, p. 64

35. DESHMUKH, M.B., DESHMUKH, D.S., Indian. J. Heterocycl. Chem., 3, 1994, p. 207

36. CLAESEN, M., VANDERHAEGHE, H., Bull. Soc. Chim. Belg., 68, 1959, p. 220

37. CRAWFORD, M., LITTLE, W.T., J. Chem. Soc., 1959, p. 729

38. Clinical and Laboratory Standards Institute. Performance standards for antimicrobial susceptibility testing; Twenty-Fourth informational supplement, (M100-S24).Wayne, USA, 1, 2014, p. 34

Manuscript received: 2.05.2019 